\title{
Vibration Response of the Planetary Gears with a Float Sun Gear and Influences of the Dynamic Parameters
}

\author{
Yinghui Liu, ${ }^{1}$ Dong Zhen $\mathbb{D}^{1},{ }^{1}$ Huibo Zhang, ${ }^{1}$ Hao Zhang, ${ }^{1}$ Zhanqun Shi, ${ }^{1}$ and Fengshou Gu ${ }^{2}$ \\ ${ }^{1}$ Tianjin Key Laboratory of Power Transmission and Safety Technology for New Energy Vehicles, \\ School of Mechanical Engineering, Hebei University of Technology, Tianjin 300401, China \\ ${ }^{2}$ Centre for Efficiency and Performance Engineering, University of Huddersfield, Huddersfield HD1 3DH, UK \\ Correspondence should be addressed to Dong Zhen; d.zhen@hebut.edu.cn
}

Received 9 May 2020; Revised 15 June 2020; Accepted 1 July 2020; Published 4 August 2020

Academic Editor: Muhammad Irfan

Copyright (c) 2020 Yinghui Liu et al. This is an open access article distributed under the Creative Commons Attribution License, which permits unrestricted use, distribution, and reproduction in any medium, provided the original work is properly cited.

Planetary gearboxes are widely used in mechanical transmission systems due to their large transmission ratio and high transmission efficiency. In a planetary gearbox, the sun gear is usually set to float to balance the sharing of loads among planet gears. However, this floating set will result in the variation of pressure angle, overlap ratio, and meshing phase in the meshing progress and when gear faults occur, the variation will be enlarged. In the previous studies, these parameters were reduced to constant. To study the influence of the dynamic parameters on the vibration response of planetary gearboxes under different operating conditions, a new lumped-parameter model containing the time-varying pressure angle (TVPA), time-varying overlap ratio (TVOR), and time-varying meshing phase (TVMP) is established. Based on this model, the vibration response mechanism of the sun gear is analyzed. Moreover, the comparison with the previous model is made and the rule of phase modulation caused by these dynamic parameters is revealed. By comparing the dynamic responses under different loads and rotation speeds, the phase modulation is studied in detail. Finally, the sun gear fault is introduced, and the phase modulation is analyzed in different fault degrees. This study can provide theoretical reference for the condition monitoring and fault diagnosis of planetary gearbox based on vibration analysis.

\section{Introduction}

Nowadays, planetary gearbox is widely used in various mechanical systems, such as automobiles, helicopters, wind turbines, and robots for its advantages of large transmission ratio, high transmission efficiency, and compact structure [1]. Compared with the fixed shaft gearbox, planetary gearbox has more complex mechanical structure and gear movement, especially the time-varying vibration transfer paths, which leads to unnecessary modulation and asymmetric frequency sidebands in addition to a lot of noise in its vibration response [2-5]. Hence, for planetary gearboxes, the vibration suppression, noise reduction, and fault diagnosis become extremely difficult [6]. To effectively solve these problems, it is necessary to build a planetary gearbox model and study the mechanism of vibration.

With the development of high-speed and heavy power rotating machinery, numerous researches on gear material selection [7], gear contact analysis [8], and gear models, including uncertain model [9-11] and deterministic model, have been conducted for the studies of vibration mechanism. In 1994, Kahraman [12] proposed a nonlinear dynamic model. Later, Inalpolat and Kahraman $[13,14]$ introduced manufacturing error and time-varying meshing stiffness into the model and studied the modulation sidebands of planetary gears. Zhai et al. [15] considered the installation error of the carrier and analyzed the dynamic characteristics in different error directions. Lin et al. [16] and Parker et al. [17] studied the natural frequencies and vibration modes and gave the mathematical expression for each mode. Combined with FEM, Concli et al. [18] proposed a lumped-parameter model with 18 degrees of freedom to study the frequency characteristics of the planetary gear system. Noticing that the multisource vibration components in the vibration signals were affected by the vibration transfer paths, a dynamic model containing time-varying vibration transfer paths was 
developed [19-21]. But all of these models are single state. In light load transmission, teeth separation and back-side tooth contact may occur. Therefore, Yang et al. [22] and Shi et al. [23] proposed a two-state model by introducing gear backlash to research meshing state and vibration characteristics of the system. Zhang et al. [24] proposed a dynamic model with a multiclearance coupling to analyze the vibration characteristics. Zhao et al. [25] developed a nonlinear dynamic model containing time-varying meshing stiffness, damping, static transmission error, and backlash. In a planetary gearbox, the sun gear is usually set to float to balance the load sharing among the planetary gears. Singh [26] provided a physical explanation for the basic mechanism causing the unequal load sharing phenomenon in both floating and nonfloating systems. Kahraman [12] predicted the dynamic load sharing factor and analyzed the influence of manufacturing errors, assembly changes, and key design parameters on it. When gear failure occurs, the vibration response of the planetary gearbox will also change. Li et al. [27] analyzed the frequency components of planetary gearbox in the healthy and fault states and explained the asymmetry of frequency sidebands. Parra and Vicuña [28] studied the frequency contents of planetary gearbox vibration signal via comparing phenomenological model with lumped-parameter model under nonfault and different fault conditions. Feng and Zuo [29] added the gear fault into the model to analyze the amplitude modulation and frequency modulation spectrum characteristics of the vibration signal. For early fault detection, Liu et al. [30] proposed a resultant vibration signal model and analyzed the faulty features of a signal stage planetary gearbox under nonstationary load conditions. Chen and Shao [31] analyzed the influence of tooth root crack at different positions and inclination angles on the dynamic response of the planetary gear system. Parker et al. [32,33] pointed out that the meshing phase had an important influence on the static and dynamic response of planetary gearbox and gave the meshing phase relationship among planet gears, sun gear, and ring gear. Later, Peng et al. $[34,35]$ applied meshing phase information to diagnose fault of planetary gearbox.

However, due to the sun gear floating set, bearing elastic deformation, and gear faults, the pressure angle and overlap ratio of the meshing gears will continually change in the operating process, and these variations are rarely considered in the previous established model. To study the influence of time-varying pressure angle and overlap ratio on the vibration response, a dynamic model with a floating sun gear is established. In this model, the TVPA, TVOR, and TVMP were considered as well as the vibration transfer path and time-varying meshing stiffness. Based on this model, the vibration response mechanism of the sun gear is analyzed. And then by adding typical sun gear fault, the influences of the fault on TVPA, TVOR, TVMP, and dynamic response are investigated. By comparing with the previous model under different operating conditions, the phase modulation rule is also analyzed.

The rest of this paper is organized as follows. Section 2 establishes the dynamic model of the planetary gearbox. In Section 3, the variation rule and phase modulation rule of time-varying pressure angle and overlap ratio under different working conditions and different fault severity are analyzed based on the proposed dynamic model. And the conclusions are given in Section 4.

\section{Dynamic Model of the Planetary Gearbox}

2.1. Parameter Definition. The dynamic model of the gearbox in this study is a $2 \mathrm{~K}-\mathrm{H}$ planetary gear model which is displayed in Figure 1. This model contains a sun gear (s), $n$ identical planet gears $\left(p_{i}, i=1,2, \ldots n\right)$, a ring gear $(r)$, and a carrier (c). The carrier serves as power input component and the sun gear is connected to the output shaft. All gear bodies are regarded as rigid and each element has one rotational and two translational degrees of freedom [12]. The coordinate systems used in this model are illustrated in Figure 2. The absolute coordinate system XOY is fixed on the ring gear, and the rotating frame of reference $\{u, v\}$ is attached to the carrier. The translational displacements of the sun gear, the carrier, and the ring gear are denoted as $x_{j}, y_{j}, j=s, c, r$, respectively. The translational displacements of planet gears are defined as $\tau_{p_{i}}$, $\eta_{p_{i}}, i=1,2, \ldots n$, respectively. And all these translational displacements are measured with respect to the coordinate $\{\mathrm{u}, \mathrm{v}\}$. The basis coordinate $\{u, v\}$ rotates at the speed of $\Omega_{c}$ together with the carrier. And the rotational coordinate can be expressed as $u_{j}=r_{j} \times \theta_{j}, j=s, c, r, p_{1}, \ldots, p_{n}$, where $r_{j}$ is the base circle radius of the sun gear, the ring gear, planet gears, and the radius of the circle passing through the planet centers for the carrier, and $\theta_{j}$ is the component rotation angle. In the model, circumferential planet locations are specified by the fixed angle $\varphi_{p_{i}}$, where $\varphi_{p_{i}}$ is measured relative to rotating basis vector $u$ so that $\varphi_{p_{1}}=0$. The mass of elements is denoted as $m_{j}$, the inertia moment is $I_{j}$, and the number of teeth is $z_{j}(j=s, c, r$, $\left.p_{1}, \ldots, p_{n}\right)$. The supporting stiffness and damping of each element are denoted as $k_{j}$ and $c_{j}$. The gears interact with each other through the mesh stiffness of $k_{s p_{i}}$ and $k_{r p_{i}}\left(k_{s p_{i}}\right.$ for $s-p_{i}$ mesh and $k_{r p_{i}}$ for $r-p_{i}$ mesh) and mesh damping of $c_{s p_{i}}$ and $c_{r p_{i}}$ ( $c_{s p_{i}}$ for $s-p_{i}$ mesh and $c_{r p_{i}}$ for $r-p_{i}$ mesh) on the meshing action plane [12]. To simplify the model, the torsional stiffness of the shafts is neglected and the housing is regarded as rigid [25]. To reduce the asymmetry of load distribution on the planets, the sun gear is set to elastic float. The methodology followed in this study is displayed in the flow chart shown in Figure 3.

There will be contacting deflection on meshed teeth when the gearbox operates. The deflection of $s-p$ mesh and $r$ $p$ mesh can be expressed as [13]

$$
\begin{aligned}
\xi_{s p_{i}}= & x_{s} \sin \psi_{s p_{i}}-y_{s} \cos \psi_{s p_{i}}-\tau_{i} \sin \alpha_{s p_{i}}-\eta_{i} \cos \alpha_{s p_{i}} \\
& +u_{s}+u_{p_{i}}-e_{s p_{i}} \\
\xi_{r p_{i}}= & x_{r} \sin \psi_{r p_{i}}-y_{r} \cos \psi_{r p_{i}}+\tau_{i} \sin \alpha_{r p_{i}}-\eta_{i} \cos \alpha_{r p_{i}} \\
& +u_{r}-u_{p_{i}}-e_{r p_{i}}
\end{aligned}
$$

The relative deflection along $\tau_{i}$ and $\eta_{i}$ between the carrier and planet can be expressed as [13] 


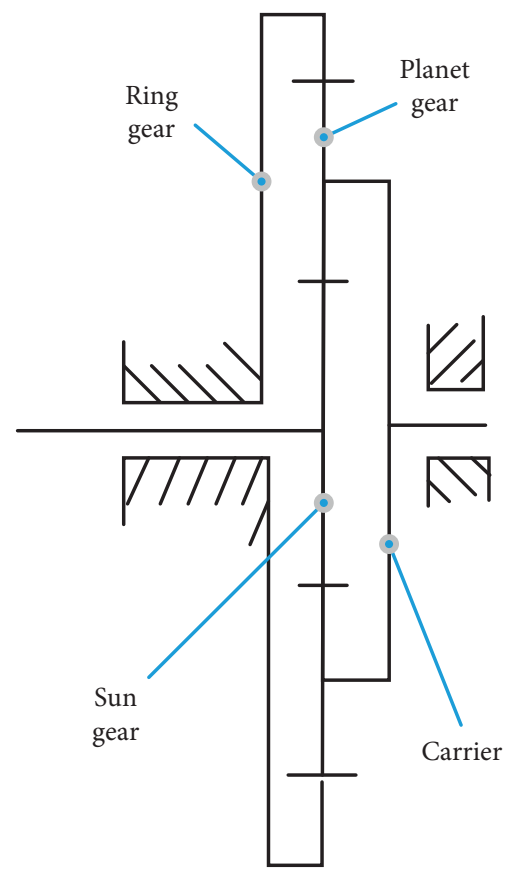

Figure 1: The structure diagram of a $2 \mathrm{~K}-\mathrm{H}$ planetary gear model.

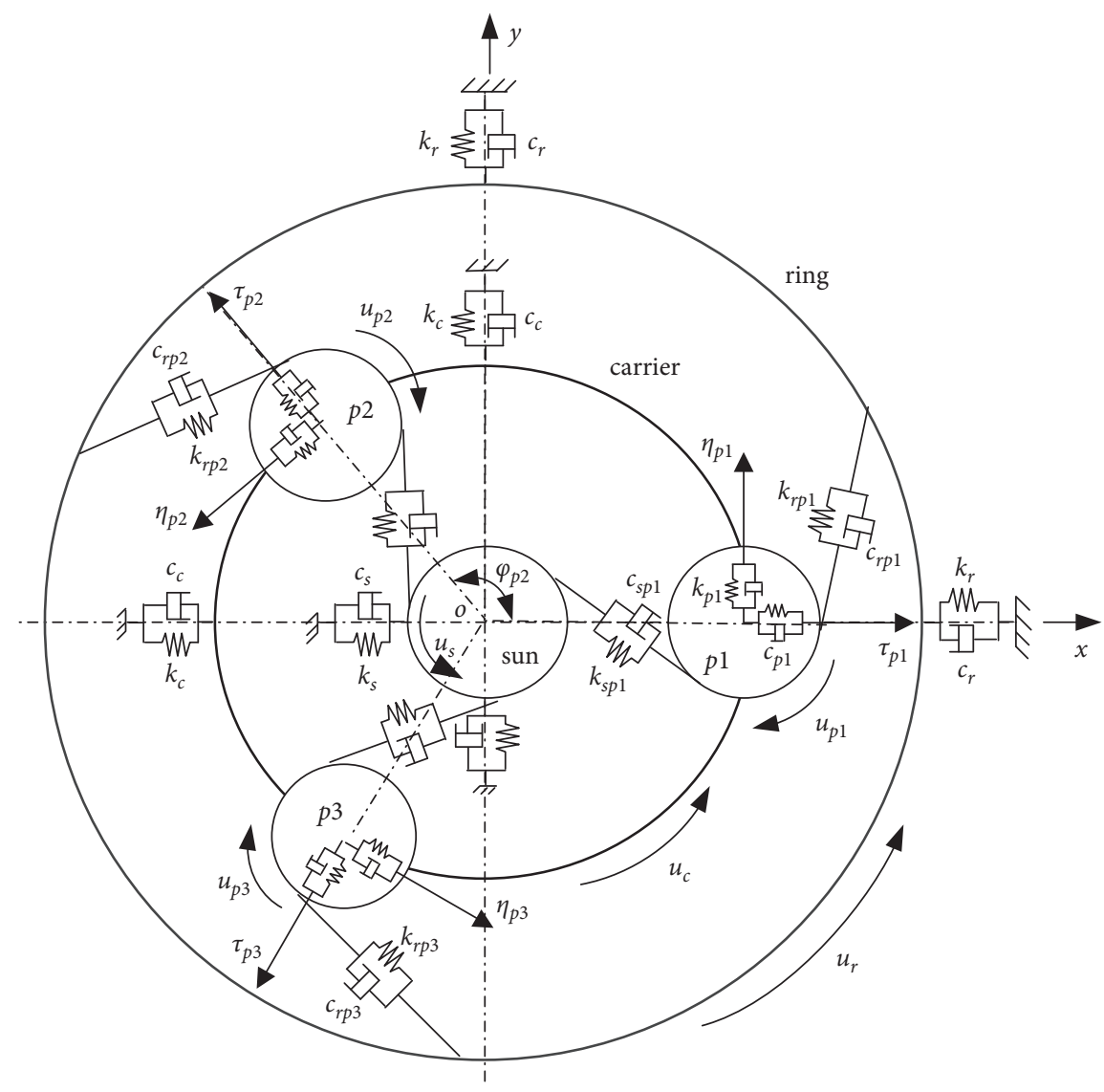

Figure 2: The dynamic model with three planet gears located at equal angle. 


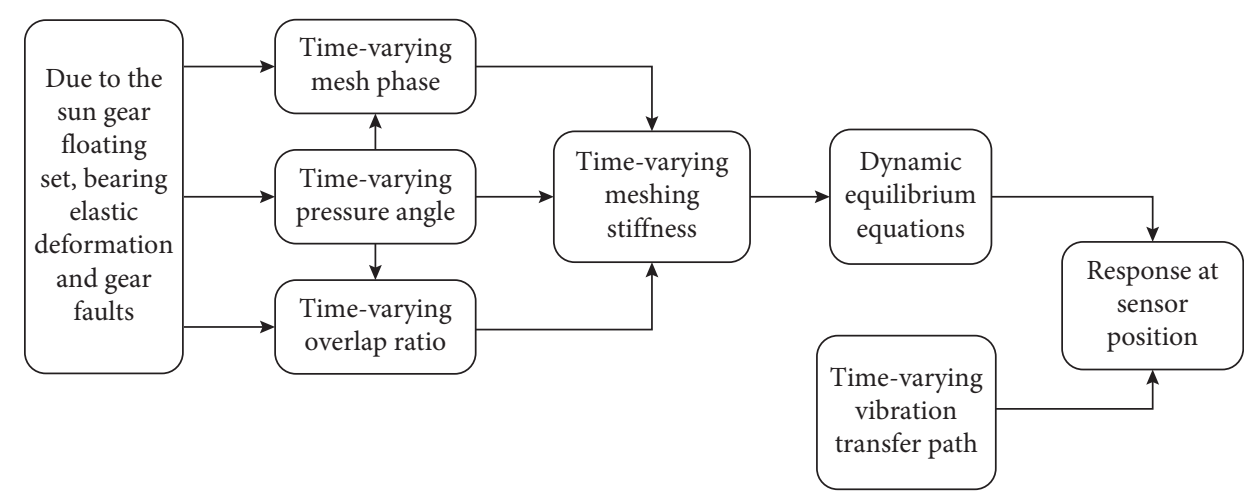

Figure 3: Flow chart of the methodology.

$$
\begin{aligned}
& \xi_{c p_{i} x}=x_{c} \cos \varphi_{p_{i}}+y_{c} \sin \varphi_{p_{i}}-\tau_{p_{i}} \\
& \xi_{c p_{i} y}=x_{c} \sin \varphi_{p_{i}}-y_{c} \cos \varphi_{p_{i}}-\eta_{p_{i}}+u_{c} .
\end{aligned}
$$

where $e_{s p_{i}}$ and $e_{r p_{i}}$ represent the transmission error of $s-p_{i}$ and $r-p_{i}$, respectively. $\psi_{s p_{i}}=\alpha_{s p_{i}}+\varphi_{p_{i}}$ and $\psi_{r p_{i}}=\alpha_{r p_{i}}-\varphi_{p_{i}}$, with $\alpha_{s p_{i}}$ and $\alpha_{r p_{i}}$ denoting the pressure angle of $s-p_{i}$ and $r-p_{i}$, respectively. Because of the vibration of the floating sun gear and planet gears during the rotation of the gearbox, the pressure angles $\alpha_{s p_{i}}$ and $\alpha_{r p_{i}}$ are time-varying. And the TVPA of $s-p_{i}$ and $r-p_{i}$ can be deduced from geometry as follows [36]:

$$
\begin{aligned}
\alpha_{s p_{i}} & =\cos ^{-1} \frac{r_{s}+r_{p_{i}}}{\sqrt{\left(x_{s}-x_{p_{i}}\right)^{2}+\left(y_{s}-y_{p_{i}}\right)^{2}}}, \\
\alpha_{r p_{i}} & =\cos ^{-1} \frac{r_{r}-r_{p_{i}}}{\sqrt{\left(x_{r}-x_{p_{i}}\right)^{2}+\left(y_{r}-y_{p_{i}}\right)^{2}}},
\end{aligned}
$$

where $x_{s}$ and $y_{s}$ represent the location of the sun gear center and $x_{r}$ and $y_{r}$ denote the location of the ring gear. All these coordinates are measured in the absolute coordinate.

$x_{p_{i}}$ and $y_{p_{i}}$ are the location of the planet gear center in the absolute coordinate which can be calculated as follows [36]:

$$
\begin{aligned}
& x_{p_{i}}=x_{c}+\left(\tau_{p_{i}}+r_{c}\right) \cos \left(\theta+\varphi_{p_{i}}\right)-\eta_{p_{i}} \sin \left(\theta+\varphi_{p_{i}}\right), \\
& y_{p_{i}}=y_{c}+\left(\tau_{p_{i}}+r_{c}\right) \sin \left(\theta+\varphi_{p_{i}}\right)+\eta_{p_{i}} \cos \left(\theta+\varphi_{p_{i}}\right),
\end{aligned}
$$

where $x_{c}$ and $y_{c}$ are the position of the carrier in the absolute coordinate. $\tau_{p_{i}}$ and $\eta_{p_{i}}$ are the location of $p_{i}$ in the coordinate $\left\{\tau_{p_{i}}, \eta_{p_{i}}\right\} . \theta$ represents the rotation angle of the carrier.

And as shown in Figure 4, the variation of the meshing phase at the pitch point can be deduced as follows

$$
\begin{aligned}
\beta_{\text {start }} & =\cos ^{-1} \frac{r_{s d} \times \cos \alpha}{r_{a s}}, \\
d_{1} & =\sqrt{r_{a s}^{2}-r_{s}^{2}}+\sqrt{r_{a p}^{2}-r_{p}^{2}}-\left(r_{s}+r_{p}\right) \tan \alpha, \\
r_{\text {end }} & =\sqrt{\left(\sqrt{r_{a s}^{2}-r_{s}^{2}}-d_{1}\right)^{2}+r_{s}^{2},}
\end{aligned}
$$

$$
\begin{aligned}
\beta_{\text {end }} & =\cos ^{-1} \frac{r_{s d} \times \cos \alpha}{r_{\text {end }}}, \\
\gamma_{\text {evolve_cycle }} & =\left\{\tan \left(\beta_{\text {start }}\right)-\beta_{\text {start }}\right\}-\left\{\tan \left(\beta_{\text {start }}\right)-\beta_{\text {end }}\right\}, \\
\gamma_{\text {varyevolve_s } p_{i}} & =\left\{\tan \left(\alpha_{s p_{i}}\right)-\alpha_{s p_{i}}\right\}-\{(\tan \alpha)-\alpha\},
\end{aligned}
$$$$
\gamma_{\text {varyevolve_r } r p_{i}}=\left\{\tan \left(\alpha_{r p_{i}}\right)-\alpha_{r p_{i}}\right\}-\{(\tan \alpha)-\alpha\},
$$$$
\delta_{s p_{i}}=2 \pi \frac{\gamma_{\text {varyevolve_s } p_{i}}}{\gamma_{\text {evolve_cycle }}},
$$$$
\delta_{r p_{i}}=2 \pi \frac{\gamma_{\text {varyevolve_ } r p_{i}}}{\gamma_{\text {evolve_cycle }}} .
$$

Herein $d_{1}$ describes the length of the actual line of mesh. $\beta_{\text {start }}$ stands for the pressure angle of addendum circle of the sun gear and $\beta_{\text {end }}$ is the pressure angle for $s$ $p_{i}$ at the mesh separation point as shown in Figure 4 . $r_{\text {end }}$ is the radius of the circle for the sun gear where the mesh separation point is located. $\gamma_{\text {evolve_cycle }}$ denotes the evolving angle of the meshing tooth surface. $\gamma_{\text {varyevolve_s } p_{i}}$ and $\gamma_{\text {varyevolve_r }} p_{i}$ represent the variations of evolving angle relative to the evolving angle in the reference circle. $\delta_{s p_{i}}$ and $\delta_{r p_{i}}$ are the variations of meshing phase of $s-p_{i}$ and $r-p_{i}$ relative to the standard meshing phase whose value is $2 \pi f_{m}\left(f_{m}\right.$ is the meshing frequency).

Therefore, the actual meshing phase is $\left(\delta_{s p_{i}}+2 \pi f_{m}\right)$ for $s-p_{i}$ mesh, and the actual meshing phase is $\left(\delta_{r p_{i}}+2 \pi f_{m}\right)$ for $r-p_{i}$ mesh. $\alpha$ is the standard pressure angle of reference circle. For the gear with a reference circle pressure angle of $20^{\circ}$ is most widely used in standard gears, the value of $\alpha$ is set to $20^{\circ}$. For conjugate tooth profile, although their tooth profile shape is different in unit time, they change the same meshing phase.

For the mating gears, the meshing stiffness is not always the standard value in the progress of rotation. It changes periodically as shown in Figure 5. In one mesh cycle, when the mating gears are just coming into mesh, 


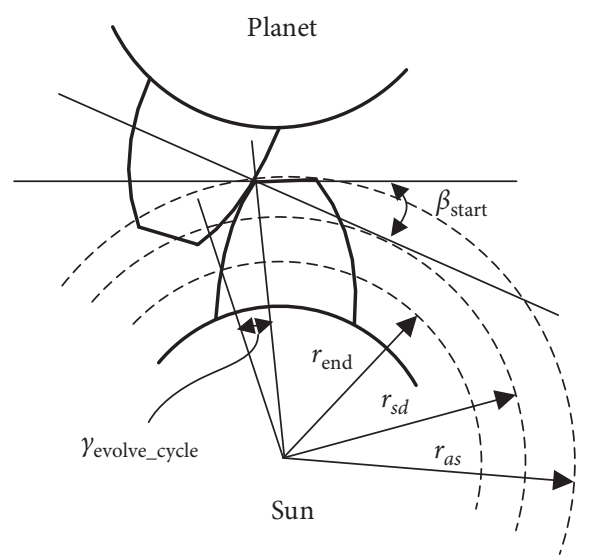

(a)

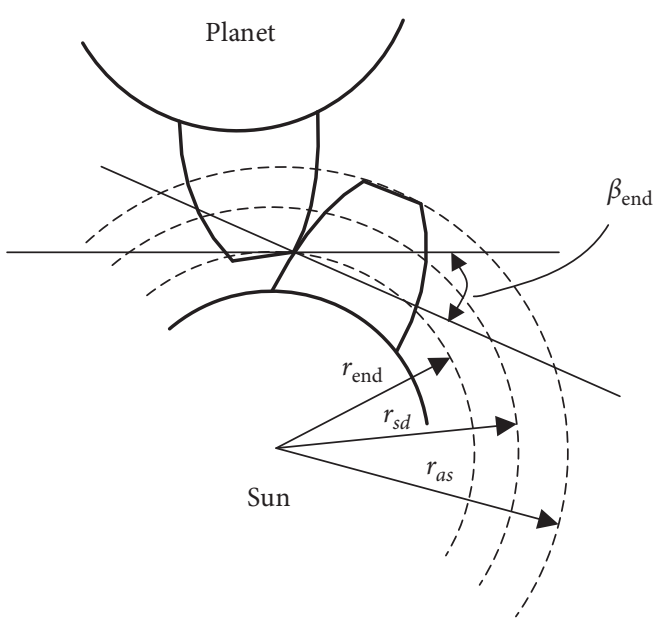

(b)

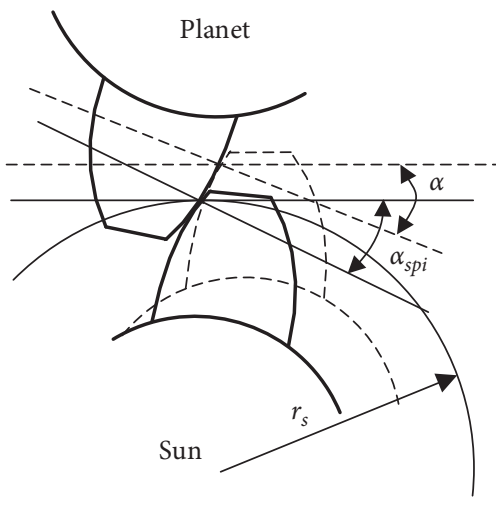

(c)

Figure 4: The meshing progress of the sun and planet gears. (a) The moment of meshing start. (b) The moment of meshing finish. (c) The meshing progress with the sun gear bounce.

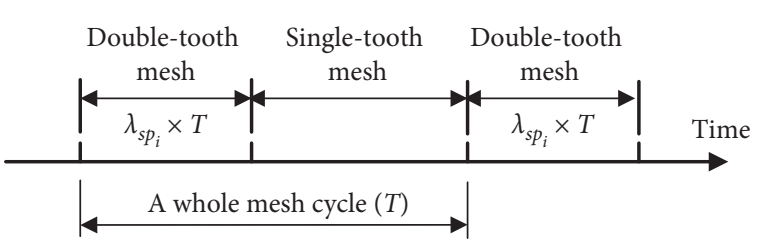

(a)

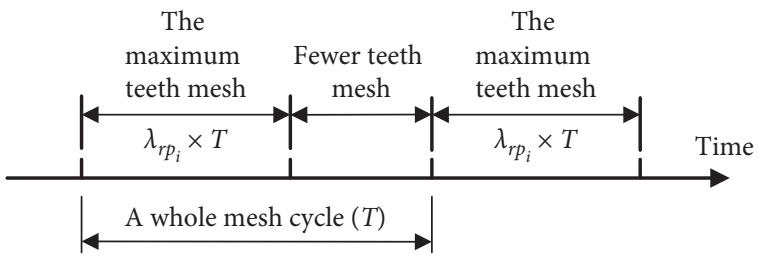

(b)

FIGURE 5: Meshing progress of one mesh cycle for the (a) external-external gear mesh and (b) internal-external gear mesh.

they are in the state of double-tooth mesh (for externalexternal mesh) or the most teeth mesh (for internalexternal mesh) and become single-tooth mesh or the fewest teeth mesh near the pitch point. When the meshing teeth are going to be separated, they come into double-tooth mesh or the most teeth mesh again. The proportion of the double-tooth mesh or the most teeth mesh in a whole mesh cycle is determined by contact overlap ratio. However, the contact overlap ratio is also time-varying, which can be defined as follows [36]

$$
\begin{aligned}
& \varepsilon_{s p_{i}}=\frac{\sqrt{r_{a s}^{2}-r_{s}^{2}}+\sqrt{r_{a p_{i}}^{2}-r_{p_{i}}^{2}}-\left(r_{s}+r_{p}\right) \tan \alpha_{s p_{i}}}{p_{b}}, \\
& \varepsilon_{r p_{i}}=\frac{\sqrt{r_{a p_{i}}^{2}-r_{p_{i}}^{2}}-\sqrt{r_{a r}^{2}-r_{r}^{2}}+\left(r_{s}+r_{p}\right)\left(\sin \alpha_{r p_{i}} / \cos \alpha_{s p_{i}}\right)}{p_{b}},
\end{aligned}
$$


where $p_{b}$ is the base pitch. $r_{a s}, r_{a p_{i}}$, and $r_{a r}$ are the addendum radii of the sun gear, planet gear, and ring gear, respectively. $\alpha_{s p_{i}}$ and $\alpha_{r p_{i}}$ are the time-varying pressure angle of $s-p_{i}$ and $r$ $p_{i}$.

The proportion of the mesh in which the maximum number of teeth participate can be expressed as follows:

$$
\begin{aligned}
& \lambda_{s p_{i}}=\varepsilon_{s p_{i}}-\left\lfloor\varepsilon_{s p_{i}}\right\rfloor, \\
& \lambda_{r p_{i}}=\varepsilon_{r p_{i}}-\left\lfloor\varepsilon_{r p_{i}}\right\rfloor .
\end{aligned}
$$

In $12(\mathrm{a})$ and $12(\mathrm{~b}),\lfloor\cdot\rfloor$ is defined as the rounding function.

Therefore, the time-varying meshing stiffness can be expressed as

$$
\begin{gathered}
k_{s p_{i}}(t)= \begin{cases}\Gamma, & \bmod \left(\theta \times z_{r}+\varphi_{p_{i}} \times z_{s}+\delta_{s p_{i}}, 2 \pi\right) \in\left[2 \pi * \lambda_{s p_{i}}, 2 \pi\left(\varepsilon_{s p_{i}}-\lambda_{s p_{i}}\right)\right], \\
1.8 \Gamma, & \bmod \left(\theta \times z_{r}+\varphi_{p_{i}} \times z_{s}+\delta_{s p_{i}}, 2 \pi\right) \in\left[0,2 \pi * \lambda_{s p_{i}}\right],\end{cases} \\
k_{r p_{i}}(t)= \begin{cases}\Lambda, & \bmod \left(\theta \times z_{r}+\varphi_{p_{i}} \times z_{r}+\rho+\delta_{r p_{i}}, 2 \pi\right) \in\left[2 \pi * \lambda_{r p_{i}}, 2 \pi\left(\varepsilon_{r p_{i}}-\lambda_{r p_{i}}\right)\right], \\
1.8 \Lambda, & \bmod \left(\theta \times z_{r}+\varphi_{p_{i}} \times z_{r}+\rho+\delta_{r p_{i}}, 2 \pi\right) \in\left[0,2 \pi * \lambda_{r p_{i}}\right] .\end{cases}
\end{gathered}
$$

In 13(a) and $13(\mathrm{~b}), \bmod (\cdot)$ is defined as the remainder function. $\Gamma$ is the meshing stiffness constant corresponding to the single-tooth mesh area of $s-p_{i}$ and $1.8 \Gamma$ is the internalexternal mesh corresponding to the double-tooth mesh area of $s-p_{i} . \theta$ stands for the rotation angle of the carrier. Because of the vibration of gears and the TVPA, $\theta$ is not simply a linear function of time. In this model, it is calculated by numerical integral. $\varphi_{p_{i}}$ is the circumferential location angle of the planet gear. A mesh cycle can be divided into singletooth mesh and double-tooth mesh, and the whole mesh cycle is regarded as $2 \pi$ radian. The ring gear is fixed, when the carrier rotates $\theta$ radian, the meshing phase variation of meshed gears is $\times z_{r}$, and the initial phase of meshed gears is $\varphi_{p_{i}} \times z_{s}$ which is caused by the location angle of planets. The initial meshing phase of $s-p_{1}$ is zero. $\delta_{s p_{i}}$ is the variation of meshing phase relative to the theoretical meshing phase whose value is $\theta \times z_{r}+\varphi_{p_{i}} \times z_{s}$ for $s-p_{i}$ mesh and $\theta \times z_{r}+$ $\varphi_{p_{i}} \times z_{r}+\rho$ for $r-p_{i}$ mesh. $\rho$ is the initial meshing phase difference of $s-p_{i}$ and $r-p_{i}$. For different planet gears, $\rho$ has the same value. Hence, when the carrier rotates $\theta$ radian, the meshing phase of $s-p_{i}$ mesh is $\theta \times z_{r}+\varphi_{p_{i}} \times z_{s}+\delta_{s p_{i}}$, and the $r$ - $p_{i}$ mesh is $\theta \times z_{r}+\varphi_{p_{i}} \times z_{r}+\rho+\delta_{r p_{i}}$. As shown in Figure 6 , the meshing stiffness mutates at the moment that the meshing teeth divorce. This is just because of the TVPA and the TVOR.

In the actual measurement, the sensor is generally arranged on the shell of the gearbox and its position is fixed. However, due to the rotation of the carrier, the meshing points of these gears periodically approach and then move away from the sensor, which will affect the energy of vibration signals collected by the sensor. Therefore, to simulate this pass-through effect of planetary gears, the Hanning window can be used as a weighting function which can be described as [13]

$$
w=\frac{1}{2}-\frac{1}{2} \cos \left(\frac{2 \pi n t}{T_{c}}\right)
$$

For planet $p_{i}$, the weight function can be written as follows [13]:

$$
\begin{aligned}
& w_{p_{i}}=\sigma_{p_{i}} \times w\left(t-\frac{\varphi_{p_{i}} T_{c}}{2 \pi}\right), \\
& \sigma_{p_{i}}=\left\{\begin{array}{l}
1, \quad \bmod \left(t, T_{c}\right) \in\left[\frac{T_{c}}{n}(i-1), \frac{T_{c}}{n} i\right], \\
0, \quad \bmod \left(t, T_{c}\right) \notin\left[\frac{T_{c}}{n}(i-1), \frac{T_{c}}{n} i\right],
\end{array}\right.
\end{aligned}
$$

where $T_{c}$ denotes the rotation cycle of the carrier and $\bmod (\cdot)$ is defined as the remainder function.

The amplitude of vibration signal received by the sensor is calculated by the weight function when the meshing point is located in the action region. Outside the action area, the vibration energy of the meshing point has a great attenuation in the process of transferring to the sensor, so the vibration of the meshing point from the position outside the action region can be ignored. Therefore, the total acceleration measured by the sensor can be determined as follows [13]:

$$
a=\sum_{i=1}^{n}\left(S_{s p_{i}} w_{p_{i}} a_{s p_{i}}+S_{r p_{i}} w_{p_{i}} a_{r p_{i}}\right),
$$

where $S_{s p_{i}}$ and $S_{r p_{i}}$ are the attenuation coefficient of the energy during the transmission from the meshing point to the sensor. Because the transmission path for the externalexternal mesh is longer than that of the internal-external mesh, the value of $S_{s p_{i}}$ should be smaller than $S_{r p_{i}}$. In this model, they are set as $S_{s p_{i}}=0.4$ and $S_{r p_{i}}=0.7$, respectively.

2.2. Dynamic Equations. Based on the above parameter definitions, the dynamic equilibrium equations can be listed out.

For the carrier, the dynamic equations can be expressed as 


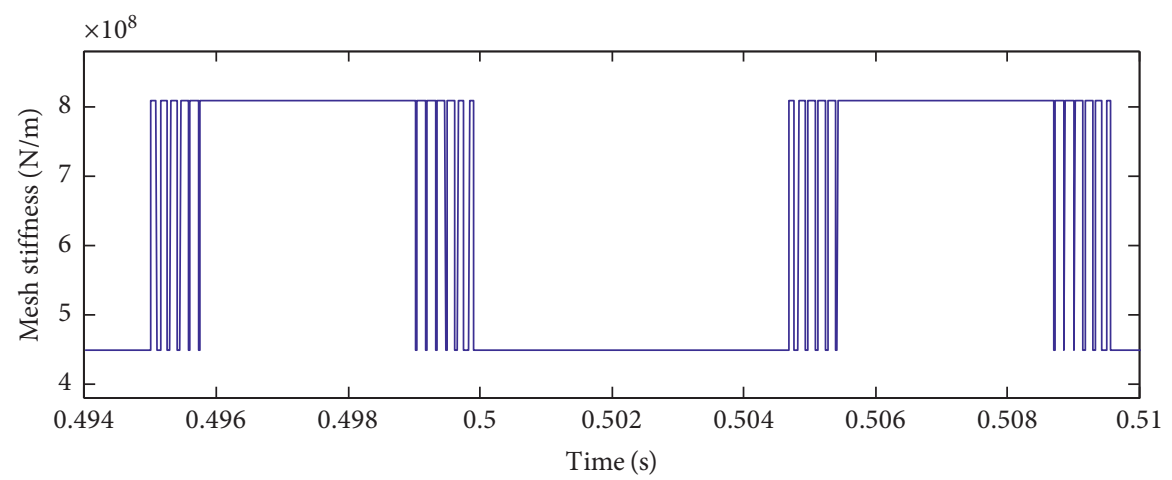

Figure 6: Meshing stiffness of $s-p_{1}$ mesh.

$$
\begin{gathered}
m_{c} \ddot{x}_{c}+k_{c} x_{c}+c_{c} \dot{x}_{c}-\sum_{i=1}^{n}\left(k_{p_{i}} \tau_{p_{i}}+c_{p_{i}} \dot{\tau}_{p_{i}}\right) \cos \varphi_{p_{i}} \\
-\sum_{i=1}^{n}\left(k_{p_{i}} \eta_{p_{i}}+c_{p_{i}} \dot{\eta}_{p_{i}}\right) \sin \varphi_{p_{i}}=0, \\
m_{c} \ddot{y}_{c}+k_{c} y_{c}+c_{c} \dot{y}_{c}-\sum_{i=1}^{n}\left(k_{p_{i}} \tau_{p_{i}}+c_{p_{i}} \dot{\tau}_{p_{i}}\right) \sin \varphi_{p_{i}} \\
+\sum_{i=1}^{n}\left(k_{p_{i}} \eta_{p_{i}}+c_{p_{i}} \dot{\eta}_{p_{i}}\right) \cos \varphi_{p_{i}}=0, \\
\frac{I_{c}}{r_{c}^{2}} \ddot{u}_{c}+\sum_{i=1}^{n}\left(k_{p_{i}} \eta_{p_{i}}+c_{p_{i}} \dot{\eta}_{p_{i}}\right)=\frac{T_{c}}{r_{c}},
\end{gathered}
$$

where $T_{c}$ is the input torque.

And the dynamic equations of the sun gear, ring gear, and planetary gears can be given referring to [37].

\section{Numerical Simulation}

3.1. Ideal Pressure Angle, Overlap Ratio, and Meshing Phase. The basic parameters used in this model are shown in Table 1 . The input speed of the carrier is $100 \mathrm{rpm}$, and the input toque is $2500 \mathrm{Nm}$. The supporting stiffness of the sun gear, ring gear, carrier, and planet gears are set up as $5 \mathrm{E} 7 \mathrm{~N} / \mathrm{m}$, $1 \mathrm{E} 8 \mathrm{~N} / \mathrm{m}, 1 \mathrm{E} 8 \mathrm{~N} / \mathrm{m}$, and $2 \mathrm{E} 9 \mathrm{~N} / \mathrm{m}$, respectively. The meshing stiffness constants $\Gamma$ and $\Lambda$ are $4.5 \mathrm{E} 8 \mathrm{~N} / \mathrm{m}$ and $5 \mathrm{E} 8 \mathrm{~N} / \mathrm{m}$, respectively. All the gears are standard in this model. The transmission errors of $s-p_{i}$ and $r$ - $p_{i}$ pairs are neglected. The dynamic differential equations of the model are solved by the numerical integration algorithm. Because the three planets can be seen as the same, the vibration responses of them are also the same except for the location angle difference. Therefore, in this study, we just consider the mesh of $s-p_{1}$.

Keeping the pressure angle, overlap ratio constant, and the meshing phase theoretical, the meshing stiffness of $s-p_{i}$ and $r-p_{i}$ is shown in Figure 7. Because the pressure angle and overlap ratio are constant, the cycle of meshing stiffness and phase difference $\varphi$ between the sun gear and planet gear is also constant with the value of $120^{\circ}$. For the same planet $p_{i}$, the phase difference $\rho$ between $s-p_{i}$ and $r-p_{i}$ is also constant. Figure 8 displays the meshing force of $s-p_{1}$, and it contains four times of carrier rotational frequency, meshing frequency, and its double frequency. Because of the planet location angle and the number of teeth of the ring gear, the spectrum is asymmetric and the amplitude of meshing frequency is suppressed to some extent [2].

\subsection{Time-Varying Pressure Angle, Overlap Ratio, and Meshing} Phase. When the TVPA, TVOR, and TVMP are introduced into the model, the dynamic response will change. Because of the floating set, the sun gear presents a triangular motion due to the meshing force of $s-p_{i}$ which is shown in Figure 9, and the center track of the sun gear is shown in Figure 10. If the sun gear and planet gears have only rotational freedom and do not consider the effect of manufacturing errors, the deflection of $\xi_{s p_{1}}, \xi_{s p_{2}}$, and $\xi_{s p_{3}}$ will have the same value during the rotation. However, the meshing stiffness of $k_{s p_{1}}$, $k_{s p_{2}}$, and $k_{s p_{3}}$ is different. As a result, the sun gear is subject to unbalanced meshing forces $F i$ from the three planet gears which can be decomposed into $F i_{x}, F i_{y}$, and $M$ as shown in Figure 9(a), and the resultant force is in the same direction as $F 1$. Therefore, the sun gear will generate a translational motion trend along the direction of $F 1$ to cause the resultant force of the meshing force $F 1, F 2$, and $F 3$ to be zero. When the meshing phase of $s-p_{i}$ changes $\varphi$ radian, the force of the sun gear is shown in Figure 9(b) and the sun gear will generate a translational motion trend along the direction of $F 2$. By the same token, when the meshing phase of $s-p_{i}$ changes another $\varphi$ radian, just like Figure 9(c), the sun gear will generate a translational motion trend along the direction of F3. This combination of translational and rotational motion of the sun gear presents a motion similar to triangle. And the triangular motion of the sun gear causes that the pressure angle and overlap ratio change in a nearly sinusoidal way whose period is the meshing cycle. And there are six turning points in one cycle which are caused by the six obvious mutations of meshing stiffness between the sun gear and three planets in one mesh cycle as shown in Figure 11. Comparing Figures 7 and 11, it can be seen that when the stiffness value changes suddenly at the critical point of meshing, the meshing pressure angle also changes suddenly. Correspondingly, there are also six turning points in one cycle of TVOR as shown in Figure 12. The meshing stiffness of $s-p_{1}$ is shown in Figure 6. Because of the TVPA and 
TABLE 1: Basic parameters of the model.

\begin{tabular}{|c|c|c|c|c|}
\hline Parameters name & Sun gear & Ring gear & Planet gear & The carrier \\
\hline Number & 1 & 1 & 3 & 1 \\
\hline Number of teeth & 10 & 62 & 26 & - \\
\hline Mass (kg) & 0.28 & 3.64 & 0.34 & 2 \\
\hline Gear module $(\mathrm{mm})$ & 2.25 & 2.25 & 2.25 & - \\
\hline Standard pressure angle $\left({ }^{\circ}\right)$ & 20 & 20 & 20 & - \\
\hline Inertia moment $\left(\mathrm{kg} \cdot \mathrm{m}^{2}\right)$ & $2.10 E-04$ & $5.05 E-04$ & $2.90 E-04$ & $4.93 E-03$ \\
\hline Fault feature frequency $(\mathrm{Hz})$ & 31 & 5 & 7.95 & - \\
\hline Rotation frequency $(\mathrm{Hz})$ & 12 & 0 & 2.31 & 1.67 \\
\hline Meshing frequency $(\mathrm{Hz})$ & 103.33 & 103.33 & 103.33 & 103.33 \\
\hline
\end{tabular}

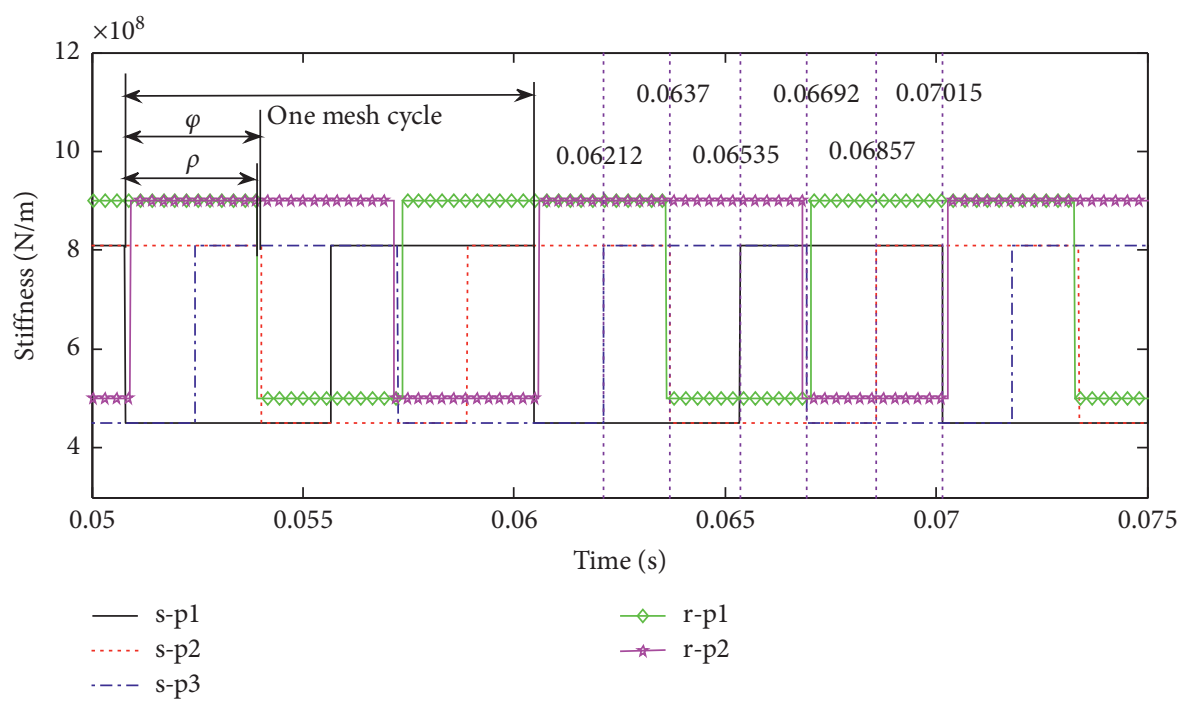

Figure 7: Meshing stiffness of different planets with the sun gear and ring gear.

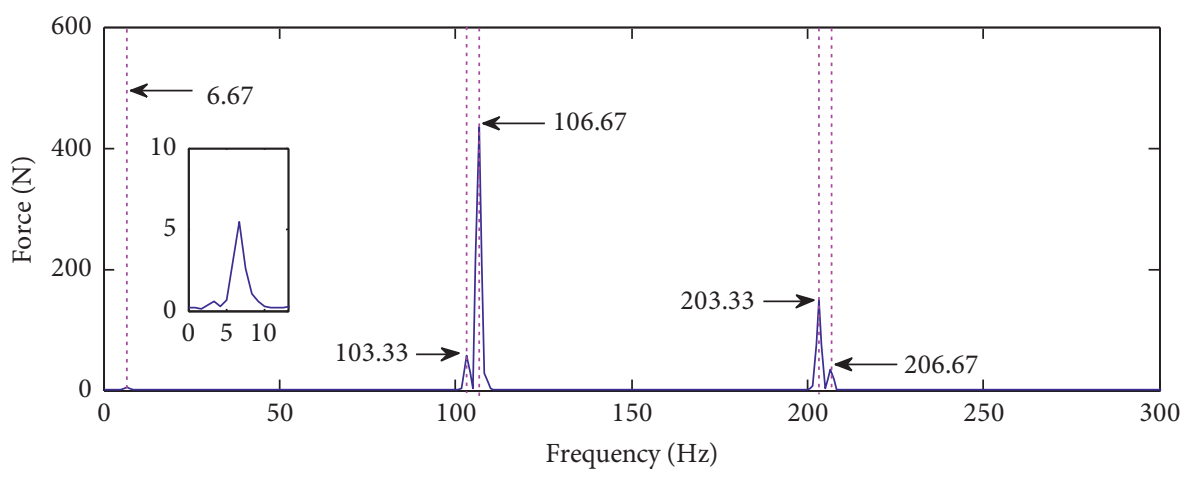

FIGURE 8: Meshing force spectrum of $s$ - $p_{1}$ mesh.

TVOR, the value of meshing stiffness result in small fluctuations on the basis of theoretical value which can be easily observed at the alternation between single-tooth and doubletooth mesh in Figure 6. Figure 13 shows the spectrum of meshing force of $s-p_{1}$ in which the meshing frequency and its multiplication can be easily observed. And we can also see that there are more frequency components in the conditions of TVPA and TVOR than that in the constant conditions. This is because TVPA causes the variation of meshing phase as shown in Figure 14. The variation of meshing phase gives rise to phase modulation that produces the additional frequency components. But the variation value is small and its influence on meshing force spectrum reflected in the amplitude and there are more sharp points in the timevarying condition.

\subsection{Comparison of Dynamic Response in Different Operating} Conditions. To investigate the influence of operating conditions on TVPA and TVMP, the simulation is conducted in different input speeds and torques.

With other conditions unchanged and the input torques being $1500 \mathrm{Nm}, 2000 \mathrm{Nm}$, and $2500 \mathrm{Nm}$, the TVPA and 


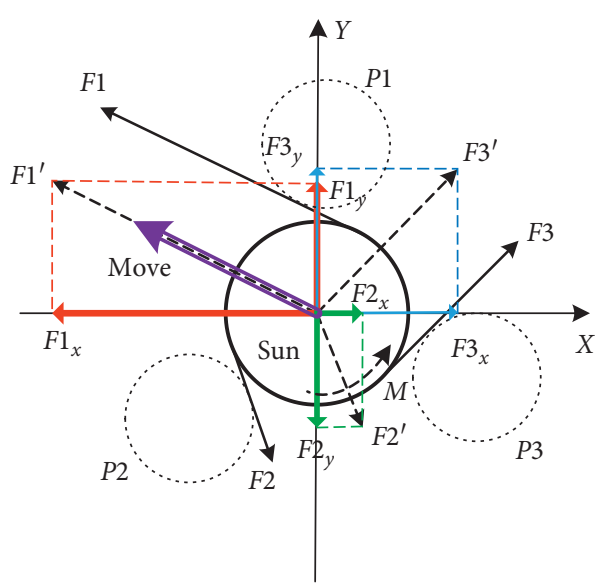

(a)

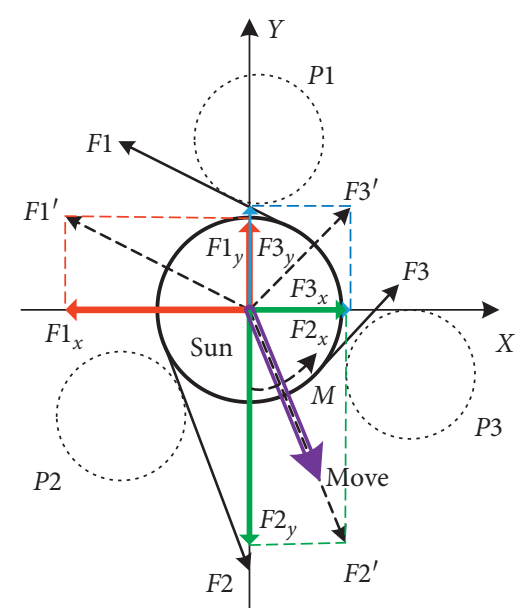

(b)

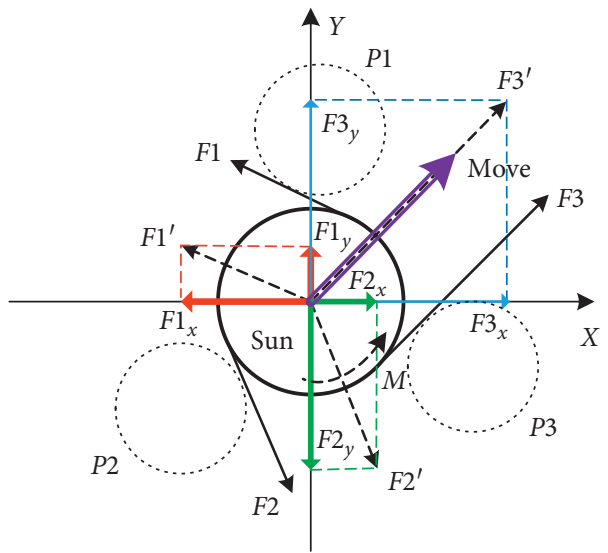

(c)

Figure 9: Diagram of meshing force on the sun gear.

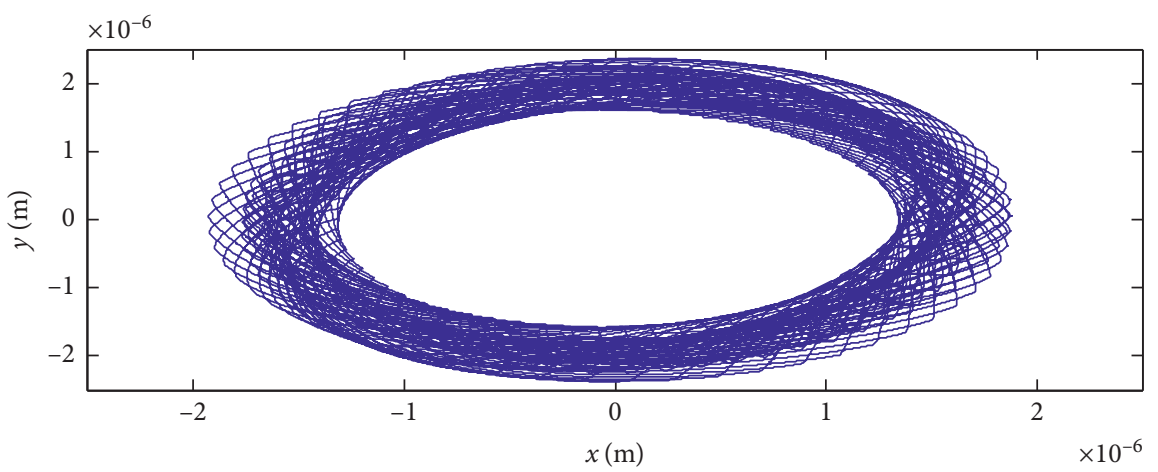

FIgURE 10: The center track of the sun gear.

TVMP of $s-p_{1}$ mesh are shown in Figures 15 and 16, respectively. With the increases of input torque, the range of variation for both of them is also increased. But the period and the form of sine is unchanged, which is nearly independent of the input torque. Also, there are six turning points in one cycle and the moment of them is almost unaffected by the different input torques. In different input torques, the pressure angle and meshing phase variation have the same amplitude modulation frequency. Figure 17 is the spectrum of the force for $s-p_{1}$ mesh in different input torques. It is clear that with the increase of input torques, the meshing force is also increased. However, due to the increases of meshing phase variation, the frequency offset will also increase, so there will be a different frequency offset in both sides of meshing frequency. In order to study the influence of phase modulation on the vibration response, the signal energy shown in Figure 18 is calculated (19999 sampling points). 


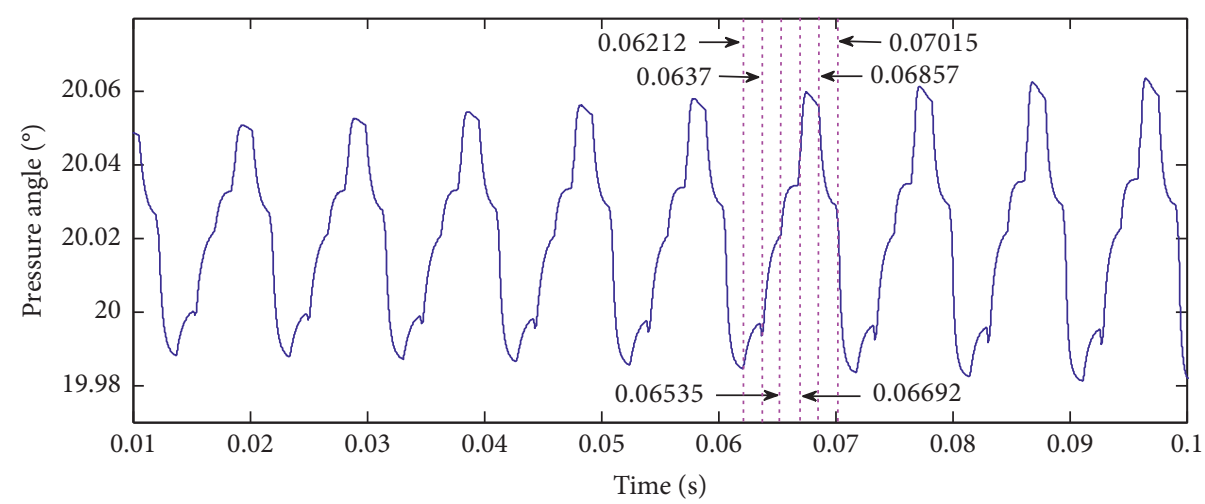

FIGURE 11: Time-varying pressure angle of $s$ - $p_{1}$ mesh.

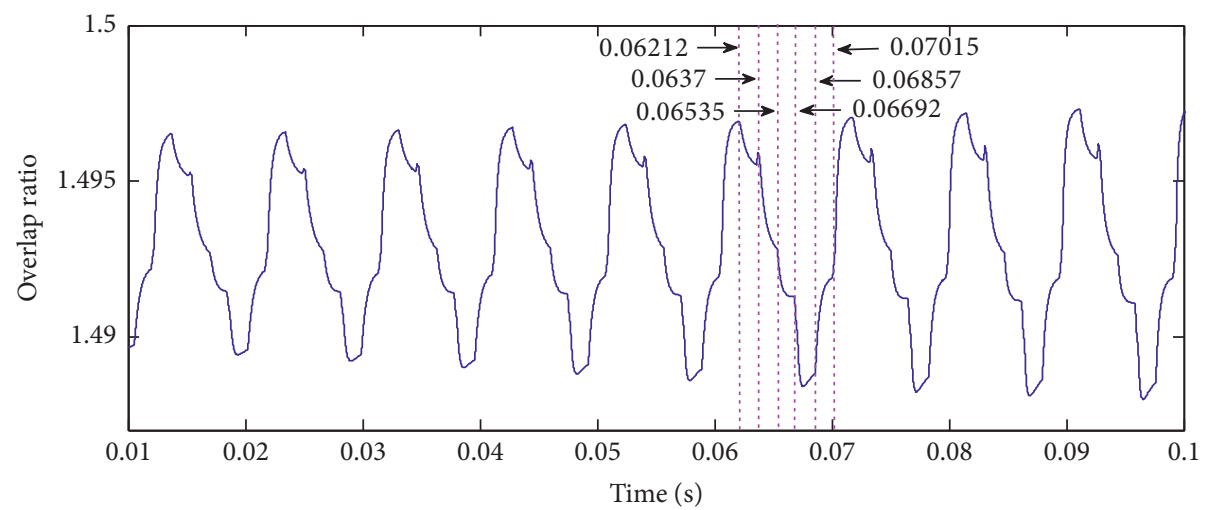

FIgURE 12: Time-varying overlap ratio of $s-p_{1}$ mesh.

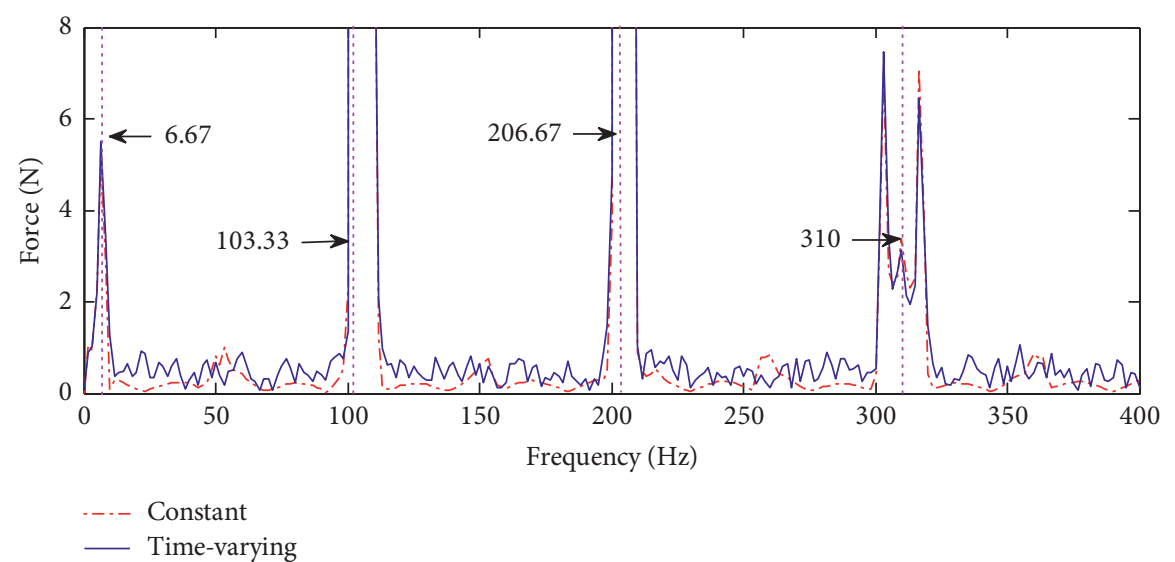

FIgURE 13: Meshing force spectrum of $s-p_{1}$ mesh in constant and time-varying pressure angle and overlap ratio.

The simulation numbers corresponding to the operation conditions are shown in Table 2. For both constant and TVPA conditions, it is calculated under the constant rotation speed and different input torques. And for the same simulation number, both of them are calculated in the same rotation speed and input torque whose energy difference is presented in Figure 19. It is obvious that as the torque increases, the signal energy is also increased linearly. This is because with the increase of torque, the amplitude of meshing vibration will also increase significantly which leads to the increase of signal energy.
However, the signal energy of constant pressure angle is larger than that of TVPA. Due to the phase modulation generated by TVPA, the amplitude of some vibrations is suppressed which makes the energy of the signal reduce. As shown in Figure 19, when the torque exceeds 2000 N/ $\mathrm{m}$, the suppression of signal vibration by phase modulation will be reduced. Therefore, it can conclude that the suppression of signal vibration by phase modulation does not always increase with the increase of torque.

With change in the input speed and the input torque being constant (input torque $=2500 \mathrm{Nm}$ ), the variations of 


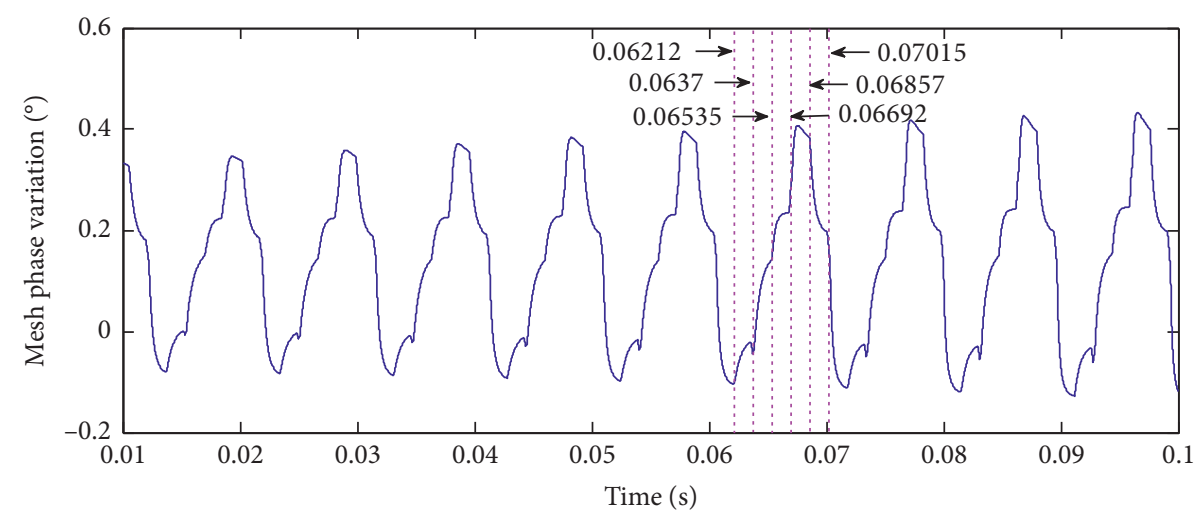

FIgURE 14: Meshing phase variation of $s$ - $p_{1}$ mesh.

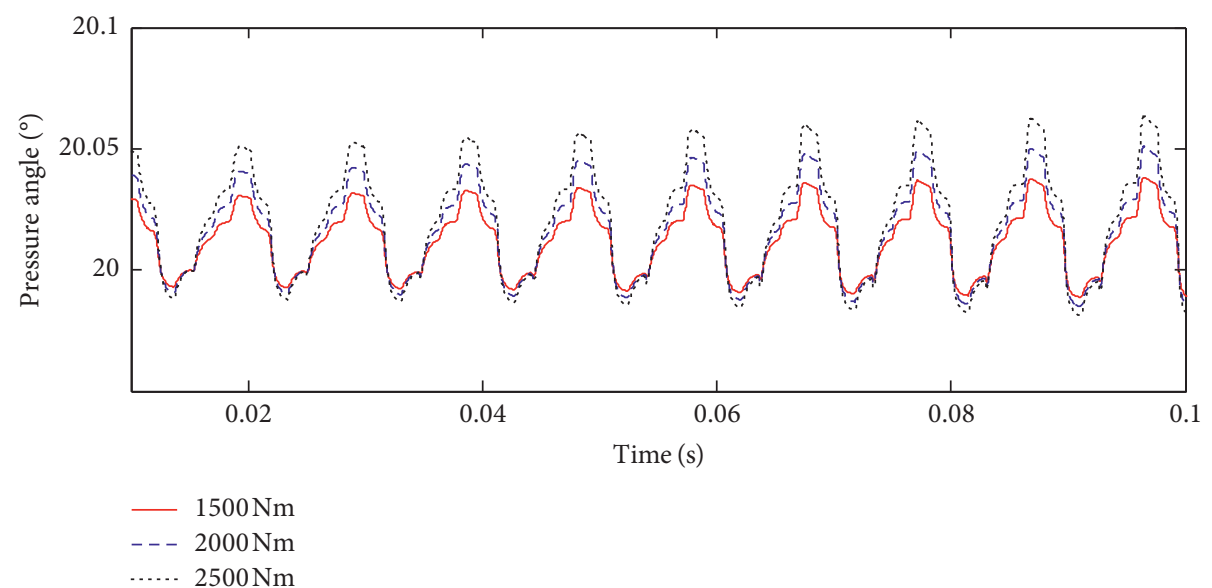

FIgURE 15: Pressure angle of $s-p_{1}$ mesh in different input torques.

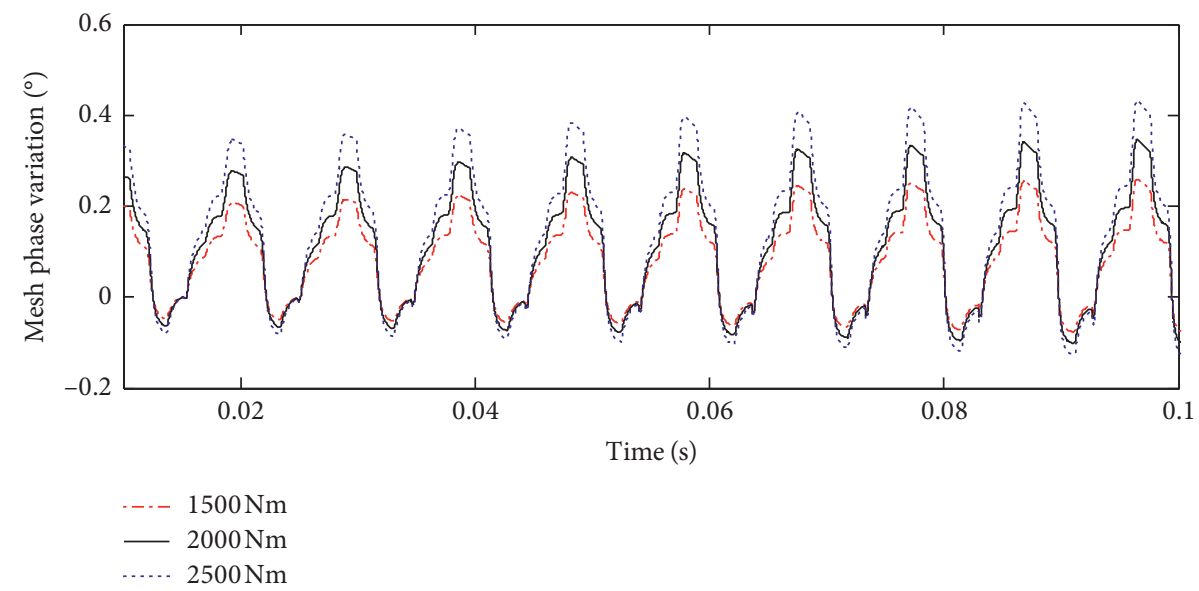

FIGURE 16: Meshing phase variation of $s-p_{1}$ mesh in different input torques.

TVPA and TVMP are shown in Figures 20 and 21. On account of the speed changes, the meshing frequency also changes. From Figures 20 and 21, it can be seen that the input speed influences both the amplitude and the period. However, the variation cycle of pressure angle equaling the meshing cycle is unchanged. And the higher the speed, the higher the variation frequency and amplitude modulation frequency will be. The energy variation of the vibration signal in different speeds is shown in Figure 22. The signal energy for both conditions of constant and TVPA is calculated in the same range of the carrier rotation angle to ensure the comparability of signal energy at different 


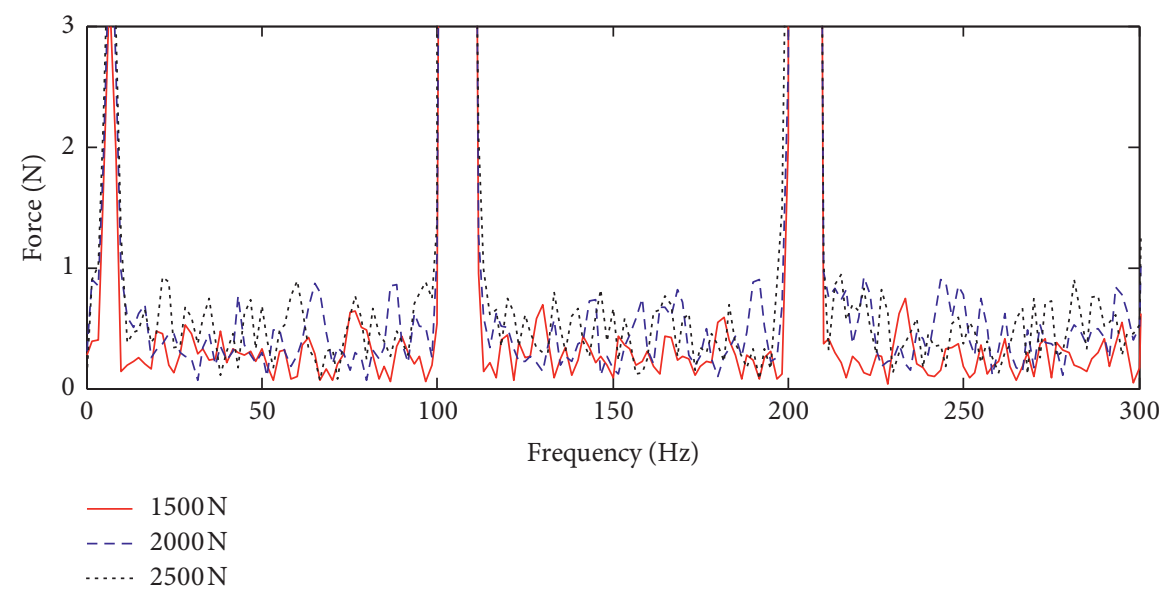

FIGURE 17: Meshing force spectrum of $s-p_{1}$ mesh in different input torques.

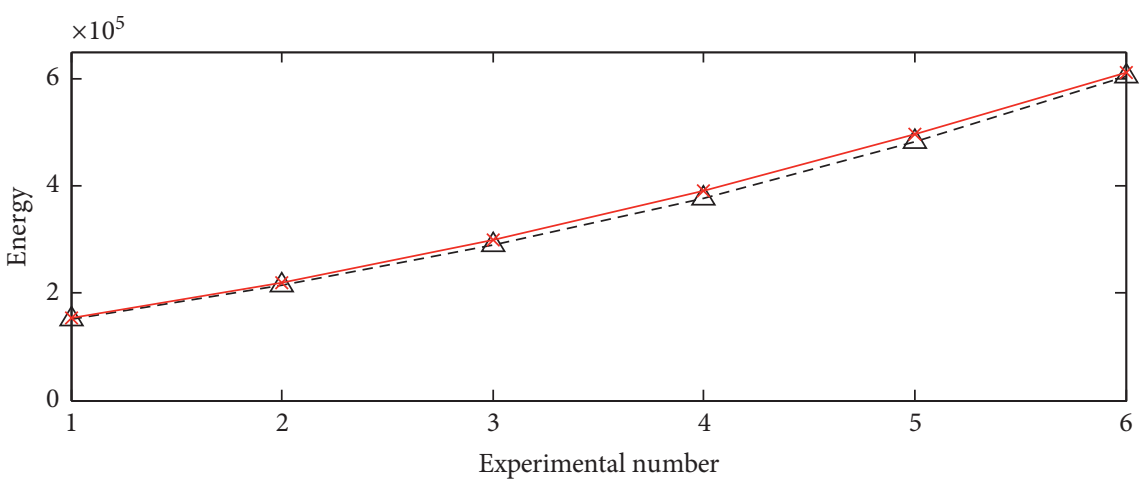

$-\triangle$ - Time-varying

$\star$ Constant

FIgURE 18: Signal energy of $s-p_{1}$ mesh in different input torques.

TABLE 2: Operating conditions.

\begin{tabular}{lcccccc}
\hline Number of operations & 1 & 2 & 3 & 4 & 5 & 6 \\
\hline Input torque $(\mathrm{Nm})$ & 1250 & 1500 & 1750 & 2000 & 2250 & 2500 \\
Input speed $(\mathrm{rpm})$ & 100 & 100 & 100 & 100 & 100 & 100 \\
\hline
\end{tabular}

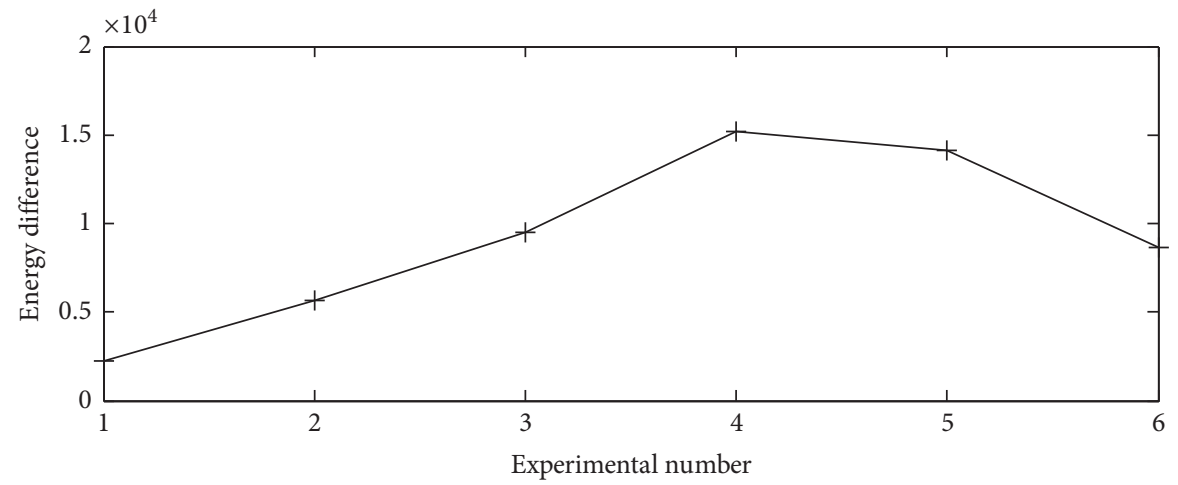

FIgURE 19: Signal energy difference between constant and time-varying conditions of $s-p_{1}$ mesh in different input torques. 


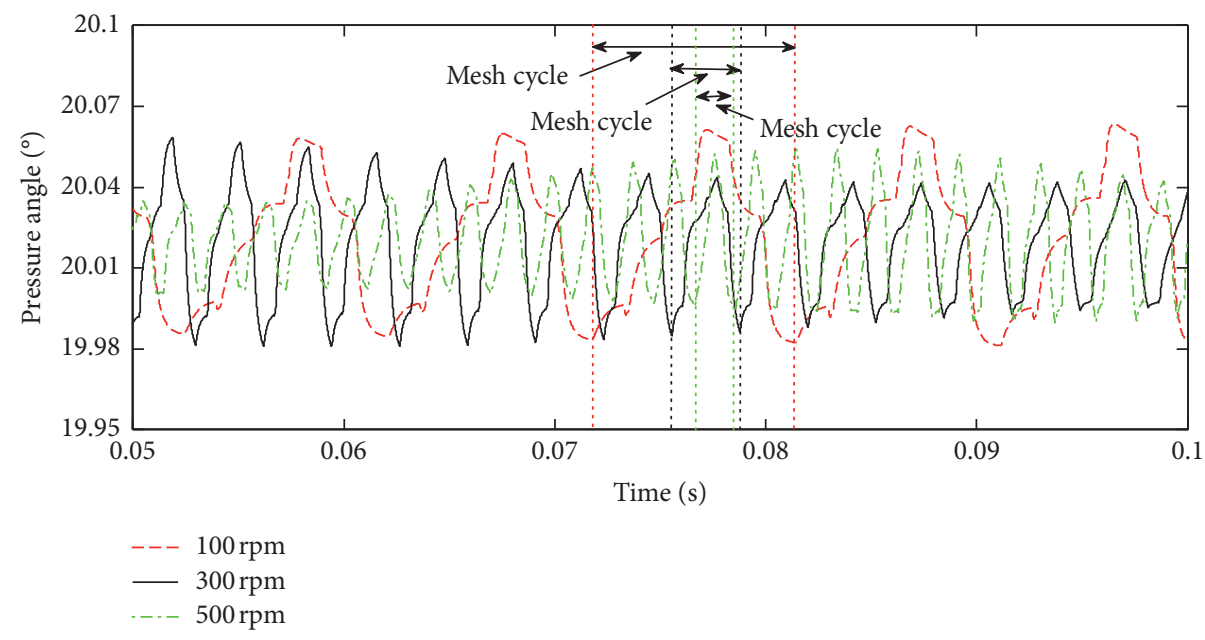

FIgure 20: Pressure angle of $s-p_{1}$ mesh in different input speeds.

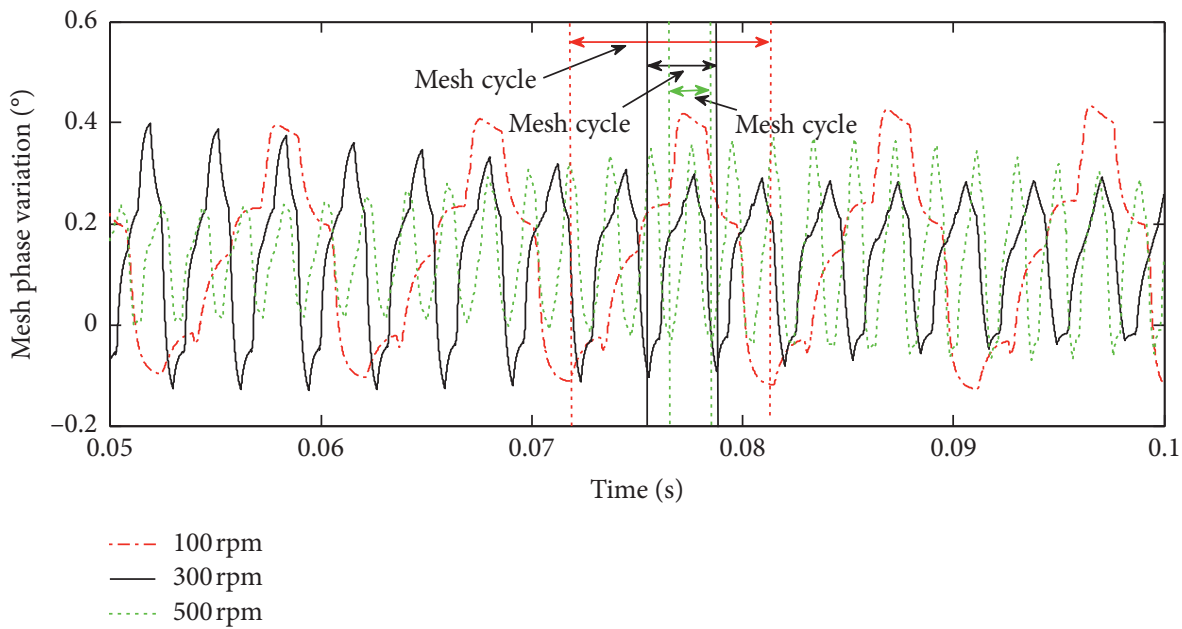

FIgURE 21: Meshing phase variation of $s-p_{1}$ mesh in different input speeds.

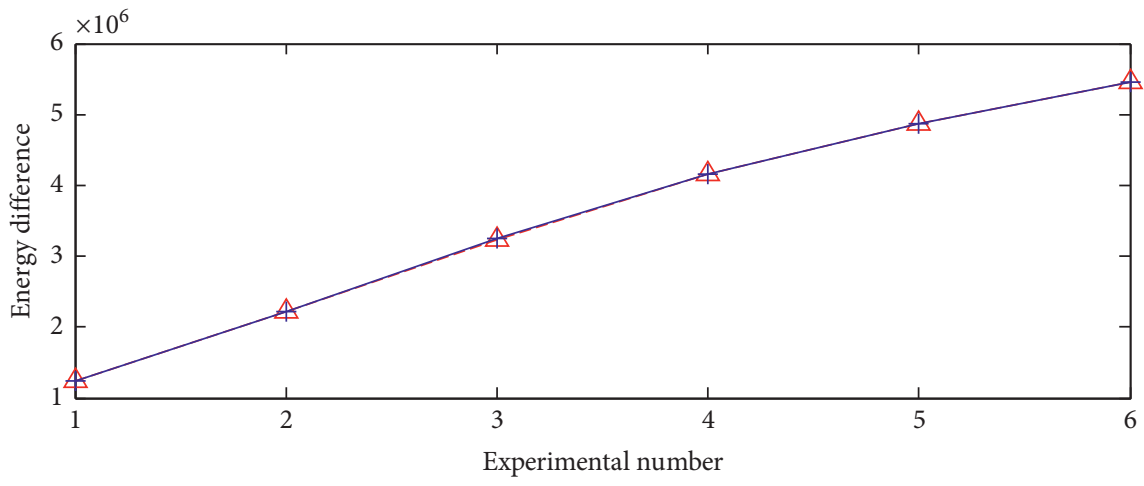

-A- Time-varying

+ Constant

Figure 22: Signal energy of $s-p_{1}$ mesh in different input speeds.

rotating speeds. The simulation numbers corresponding to the operation conditions are shown in Table 3. In Figure 22, for both of the constant and TVPA conditions, it is calculated under the constant input torque and different input speeds. And for the same simulation number, both of them are calculated in the same rotation speed and input torque whose energy difference is shown in Figure 23. From Figure 22 , it can be observed that the energy in both conditions 
TABLE 3: Operating conditions.

\begin{tabular}{lcccccc}
\hline Number of operations & 1 & 2 & 3 & 4 & 5 & 6 \\
\hline Input torque $(\mathrm{Nm})$ & 2500 & 2500 & 2500 & 2500 & 2500 & 2500 \\
Input speed $(\mathrm{rpm})$ & 100 & 200 & 300 & 400 & 500 & 600 \\
\hline
\end{tabular}

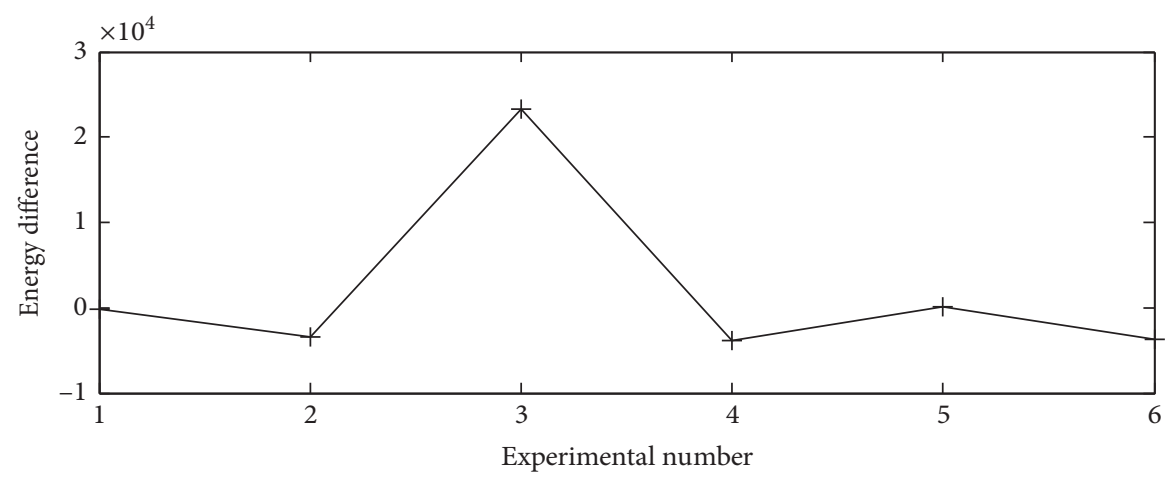

FIgURE 23: Signal energy difference between constant and TVPA conditions of $s$ - $p_{1}$ in different speeds.

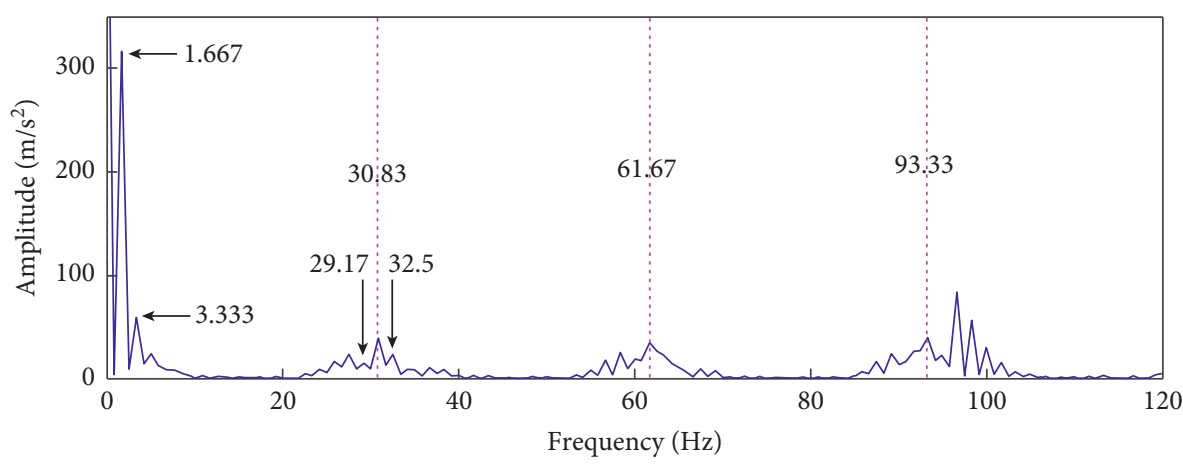

FIGURE 24: Envelope spectrum of meshing force for $s-p_{1}$ mesh in constant pressure angle.

increases linearly with the speed increasing which is because the amplitude of meshing vibration increases significantly. And at the same speed, the energy difference between the two is small except for some special speeds. Therefore, the speed variation mainly affects the phase varying period.

3.4. The Response of the System with Sun Gear Fault. To study the dynamic response of the system when a gear fault occurs, a sun gear fault is taken as an example and introduced into the model. And the fault is expressed by the attenuation of the meshing stiffness. Set the input speed $100 \mathrm{rpm}$ and the input torque $2500 \mathrm{Nm}$. The vibration signal modulates the rotation frequency of the carrier and fault characteristic frequency of the sun gear. The envelope spectrum of the meshing force for $s-p_{1}$ in a constant pressure angle is shown in Figure 24. The rotation frequency of carrier $(1.667 \mathrm{~Hz})$ and the fault characteristic frequency $(30.83 \mathrm{~Hz})$ of the sun gear can be clearly seen. And Figure 25 shows the comparison of meshing force spectrum for $s-p_{1}$ in conditions of constant pressure angle and TVPA. Due to the influence of TVPA, some frequency components in the vibration signal are strengthened and some are weakened. Therefore, the amplitude of the meshing force is not always higher than that of the constant condition, and more frequency components appear. And the response of the system under different fault severity is compared. The meshing phase variation is shown in Figure 26. The more serious the fault severity is, the greater variation of the meshing phase will be when the fault gear tooth is meshing. The variation of meshing phase is affected not only by the fault severity but also by the relative motion between the sun gear and the planet which has been explained in Section 3.2 based on Figure 8 . Therefore, the value of the meshing phase variation is not the same when the fault gear tooth and the three planets mesh, respectively, under the same fault severity. Hence the change of meshing phase will affect the phase modulation in the vibration signal and the meshing stiffness can be presented in Figure 27. It can be seen that the more serious the fault severity is, the greater the mutation value is, and so is the meshing stiffness. From the meshing force spectrum which is shown in Figure 28 , it can be concluded that with the increase of fault severity, the influence of TVPA is also strengthened. The amplitude of meshing force is different under various fault severity. 


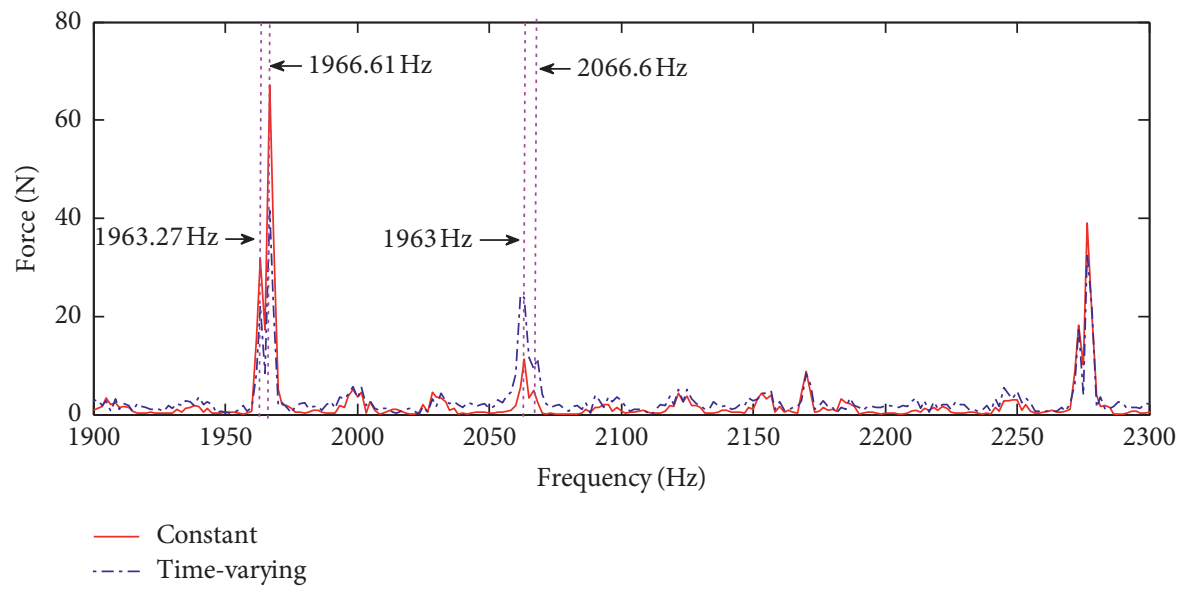

FIgURE 25: Spectral of meshing force for $s-p_{1}$ mesh in constant and time-varying pressure angle.

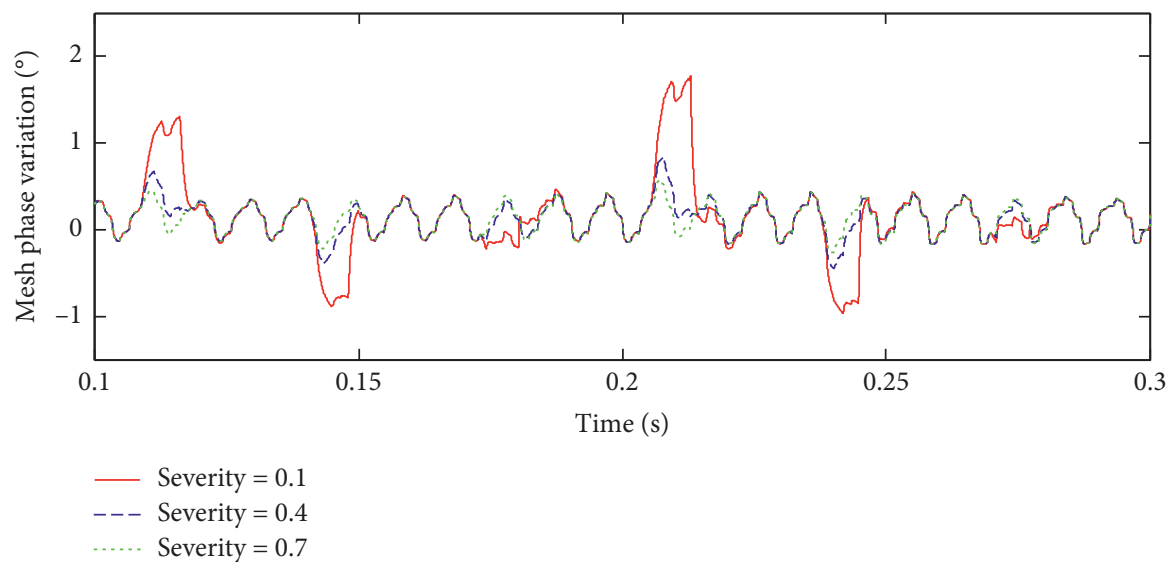

FIGURE 26: Meshing phase variation of $s-p_{1}$ mesh in different fault severity.

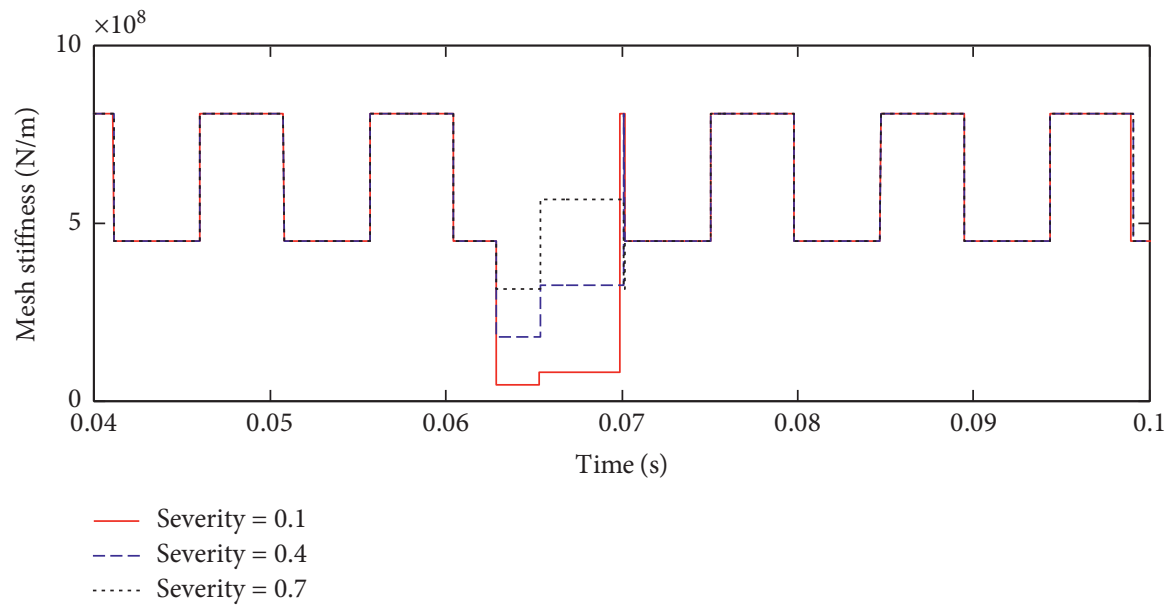

Figure 27: Meshing stiffness in different fault severity. 


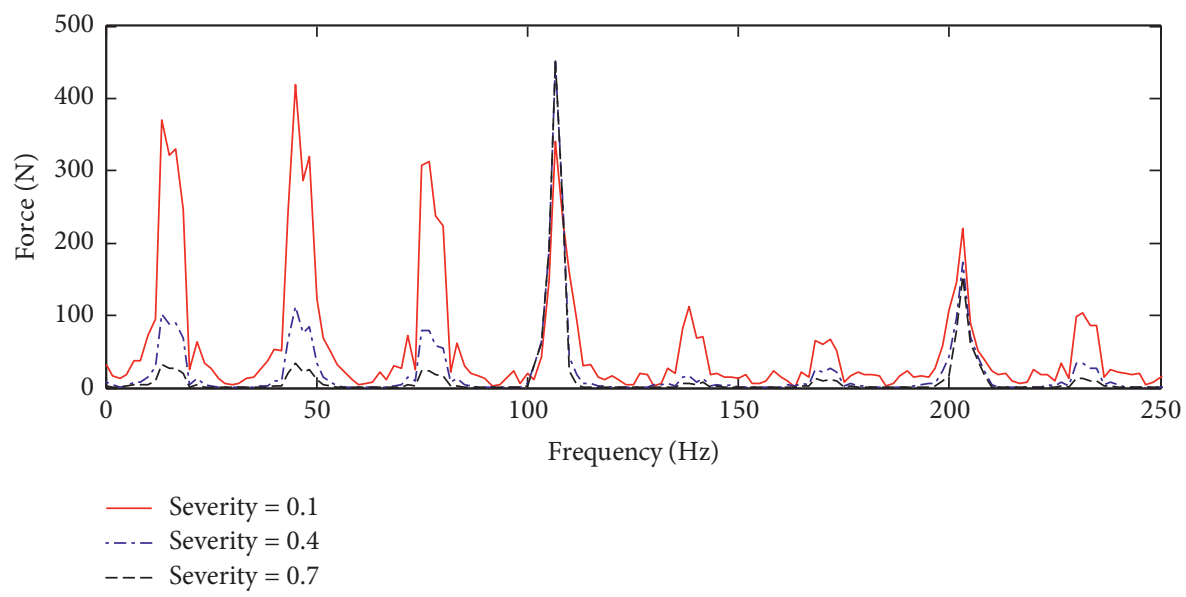

Figure 28: Meshing force of $s-p_{1}$ in different fault severity.

\section{Conclusions}

This paper studies the vibration mechanism of the sun gear when it is set to float in a planetary gearbox and introduces time-varying pressure angle, time-varying overlap ratio, and time-varying meshing phase to investigate the influence of these dynamic parameters on the vibration response of the system under different operating conditions. Subjected to the action of the meshing force, the sun gear presents a triangular movement in the mesh progress. And through analyzing the characteristics of the dynamic responses based on the proposed planetary gearbox model, the conclusions can be obtained:

(1) The time-varying pressure angle and overlap ratio vary with an approximate sine function at the period of meshing cycle, which results in more frequency components in the vibration response. Due to the floating set and bearing deflection, the pressure angle, overlap ratio, and meshing phase also change with time, which causes the meshing stiffness leading to mutation during the mesh progress.

(2) The meshing phase changes with the operating loads and rotation speeds. The rotation speed mainly affects the phase modulation period. When the load increases, the meshing phase variation will increase accordingly. Hence the phase modulation amplitude is mainly affected by the operating loads.

(3) Both the amplitude modulation and phase modulation are affected by gear faults. And when a gear fault occurs, the fault characteristic frequency will be modulated in the meshing force spectrum. The variation of time-varying pressure angle, overlap ratio, and meshing phase will increase with the fault severity.

Considering that the variation of meshing phase affects the meshing stiffness which is a main factor of gear vibration, in the future work, we will study the effect of meshing stiffness on the meshing vibration waveform to reveal the vibration mechanism, and then combine with fault feature extraction algorithm to conduct planetary gearbox fault diagnosis.

\section{Data Availability}

The datasets supporting the conclusions of this article are included within the article.

\section{Conflicts of Interest}

The authors declare that they have no conflicts of interest.

\section{Acknowledgments}

This work was supported by the National Natural Science Foundation of China (51875166, U1813222, and 51705127).

\section{References}

[1] Y. Lei, J. Lin, M. J. Zuo, and Z. He, "Condition monitoring and fault diagnosis of planetary gearboxes: a review," Measurement, vol. 48, pp. 292-305, 2014.

[2] P. D. McFadden and J. D. Smith, "An explanation for the asymmetry of the modulation sidebands about the tooth meshing frequency in epicyclic gear vibration," Proceedings of the Institution of Mechanical Engineers, Part C: Journal of Mechanical Engineering Science, vol. 199, no. 1, pp. 65-70, 1985.

[3] S. Wang, M. Huo, C. Zhang et al., "Effect of mesh phase on wave vibration of spur planetary ring gear," European Journal of Mechanics - A/Solids, vol. 30, no. 6, pp. 820-827, 2011.

[4] J. Helsen, F. Vanhollebeke, B. Marrant, D. Vandepitte, and W. Desmet, "Multibody modelling of varying complexity for modal behaviour analysis of wind turbine gearboxes," Renewable Energy, vol. 36, no. 11, pp. 3098-3113, 2011.

[5] S. T. Kandukuri, A. Klausen, H. R. Karimi, and K. G. Robbersmyr, "A review of diagnostics and prognostics of low-speed machinery towards wind turbine farm-level health management," Renewable and Sustainable Energy Reviews, vol. 53, no. 11, pp. 697-708, 2016. 
[6] G. He, K. Ding, X. Wu, and X. Yang, "Dynamics modeling and vibration modulation signal analysis of wind turbine planetary gearbox with a floating sun gear," Renewable Energy, vol. 139, pp. 718-729, 2019.

[7] A. S. Milani and A. Shanian, "Gear material selection with uncertain and incomplete data. material performance indices and decision aid model. material performance indices and decision aid model," International Journal of Mechanics and Materials in Design, vol. 3, no. 3, pp. 209-222, 2006.

[8] C. Zhou, F. Huang, X. Han, and Y. Gu, "An elastic-plastic asperity contact model and its application for micro-contact analysis of gear tooth profiles," International Journal of Mechanics and Materials in Design, vol. 13, no. 3, pp. 335-345, 2017.

[9] C. Fu, X. Ren, Y. Yang, Y. Xia, and W. Deng, "An interval precise integration method for transient unbalance response analysis of rotor system with uncertainty," Mechanical Systems and Signal Processing, vol. 107, pp. 137-148, 2018.

[10] S. Wei, Q. Han, Z. Peng, and F. Chu, "Dynamic analysis of parametrically excited system under uncertainties and multifrequency excitations," Mechanical Systems and Signal Processing, vol. 72-73, pp. 762-784, 2016.

[11] E. Wehrle, F. Concli, and L. Cortese, "Design optimization of planetary gear trains under dynamic constraints and parameter uncertainty," in Proceedings of the 8th ECCOMAS Thematic Conference on Multibody Dynamics , MBD 2017, pp. 527-538, Free University of Bozen-Bolzano, Bozen-Bolzano, Italy, January 2017.

[12] A. Kahraman, "Load sharing characteristics of planetary transmissions," Mechanism and Machine Theory, vol. 29, no. 8, pp. 1151-1165, 1994.

[13] M. Inalpolat and A. Kahraman, "A dynamic model to predict modulation sidebands of a planetary gear set having manufacturing errors," Journal of Sound and Vibration, vol. 329, no. 4, pp. 371-393, 2010.

[14] M. Inalpolat and A. Kahraman, "A theoretical and experimental investigation of modulation sidebands of planetary gear sets," Journal of Sound and Vibration, vol. 323, no. 3-5, pp. 677-696, 2009.

[15] H. Zhai, C. Zhu, C. Song, H. Liu, and H. Bai, "Influences of carrier assembly errors on the dynamic characteristics for wind turbine gearbox," Mechanism and Machine Theory, vol. 103, pp. 138-147, 2016.

[16] J. Lin and R. G. Parker, "Analytical characterization of the unique properties of planetary gear free vibration," Journal of Vibration and Acoustics, vol. 121, no. 3, pp. 316-321, 1999.

[17] R. G. Parker and X. Wu, "Vibration modes of planetary gears with unequally spaced planets and an elastic ring gear," Journal of Sound and Vibration, vol. 329, no. 11, pp. 22652275, 2010.

[18] F. Concli, L. Cortese, R. Vidoni, F. Nalli, and G. Carabin, "A mixed FEM and lumped-parameter dynamic model for evaluating the modal properties of planetary gearboxes," Journal of Mechanical Science and Technology, vol. 32, no. 7, pp. 3047-3056, 2018.

[19] Y. Lei, Z. Liu, J. Lin, and F. Lu, "Phenomenological models of vibration signals for condition monitoring and fault diagnosis of epicyclic gearboxes," Journal of Sound and Vibration, vol. 369, pp. 266-281, 2016.

[20] L. Liu, X. Liang, and M. J. Zuo, "Vibration signal modeling of a planetary gear set with transmission path effect analysis," Measurement, vol. 85, pp. 20-31, 2016.
[21] X. Liang, M. J. Zuo, and M. R. Hoseini, "Vibration signal modeling of a planetary gear set for tooth crack detection," Engineering Failure Analysis, vol. 48, pp. 185-200, 2015.

[22] T. Yang, S. Yan, and Z. Han, "Nonlinear model of space manipulator joint considering time-variant stiffness and backlash," Journal of Sound and Vibration, vol. 341, pp. 246-259, 2015.

[23] J.-f. Shi, X.-f. Gou, and L.-y. Zhu, "Modeling and analysis of a spur gear pair considering multi-state mesh with time-varying parameters and backlash," Mechanism and Machine Theory, vol. 134, pp. 582-603, 2019.

[24] H. Zhang, C. Qi, J. Fan, S. Dai, and B. You, "Vibration characteristics analysis of planetary gears with a multiclearance coupling in space mechanism," Energies, vol. 11, no. 10, p. 2687, 2018.

[25] M. Zhao and J. C. Ji, "Nonlinear torsional vibrations of a wind turbine gearbox," Applied Mathematical Modelling, vol. 39, no. 16, pp. 4928-4950, 2015.

[26] A. Singh, "Load sharing behavior in epicyclic gears: physical explanation and generalized formulation," Mechanism and Machine Theory, vol. 45, no. 3, pp. 511-530, 2010.

[27] Y. Li, K. Ding, G. He, and H. Lin, "Vibration mechanisms of spur gear pair in healthy and fault states," Mechanical Systems and Signal Processing, vol. 81, pp. 183-201, 2016.

[28] J. Parra and C. M. Vicuña, "Two methods for modeling vibrations of planetary gearboxes including faults: comparison and validation," Mechanical Systems and Signal Processing, vol. 92, pp. 213-225, 2017.

[29] Z. Feng and M. J. Zuo, "Vibration signal models for fault diagnosis of planetary gearboxes," Journal of Sound and Vibration, vol. 331, no. 22, pp. 4919-4939, 2012.

[30] X. Liu, Y. Yang, and J. Zhang, "Resultant vibration signal model based fault diagnosis of a single stage planetary gear train with an incipient tooth crack on the sun gear," Renewable Energy, vol. 122, pp. 65-79, 2018.

[31] Z. G. Chen and Y. M. Shao, "Dynamic features of a planetary gear system with tooth crack under different sizes and inclination angles," Journal of Vibration and AcousticsTransactions of the ASME, vol. 135, Article ID 031004, 2013.

[32] R. G. Parker, "A physical explanation for the effectiveness of planet phasing to suppress planetary gear vibration," Journal of Sound and Vibration, vol. 236, no. 4, pp. 561-573, 2000.

[33] R. G. Parker and J. Lin, "Mesh phasing relationships in planetary and epicyclic gears," Journal of Mechanical Design, vol. 126, no. 2, pp. 365-370, 2004.

[34] D. Peng, W. A. Smith, P. Borghesani, R. B. Randall, and Z. Peng, "Comprehensive planet gear diagnostics: use of transmission error and mesh phasing to distinguish localised fault types and identify faulty gears," Mechanical Systems and Signal Processing, vol. 127, pp. 531-550, 2019.

[35] D. Peng, W. A. Smith, R. B. Randall, and Z. Peng, "Use of mesh phasing to locate faulty planet gears," Mechanical Systems and Signal Processing, vol. 116, pp. 12-24, 2019.

[36] W. Kim, J. Y. Lee, and J. Chung, "Dynamic analysis for a planetary gear with time-varying pressure angles and contact ratios," Journal of Sound and Vibration, vol. 331, no. 4, pp. 883-901, 2012.

[37] Z. X. He, Q. T. liu, and L. H. Chang, "Transverse-torsional vibrations of the high-speed planetary gear transmission," The Open Mechanical Engineering Journal, vol. 8, no. 1, pp. 396-401, 2014. 